\title{
Generation results for vector-valued elliptic operators with unbounded coefficients in $L^{p}$ spaces
}

\section{Luciana Angiuli $^{1}$ (D) $\cdot$ Luca Lorenzi $^{2} \cdot$ Elisabetta M. Mangino ${ }^{1} \cdot$ Abdelaziz Rhandi $^{3}$}

Received: 27 April 2021 / Accepted: 3 September 2021 / Published online: 3 October 2021

(c) The Author(s) 2021

\begin{abstract}
We consider a class of vector-valued elliptic operators with unbounded coefficients, coupled up to the first order, in the Lebesgue space $L^{p}\left(\mathbb{R}^{d} ; \mathbb{R}^{m}\right)$ with $p \in(1, \infty)$. Sufficient conditions to prove generation results of an analytic $C_{0}$-semigroup $\boldsymbol{T}(t)$, together with a characterization of the domain of its generator, are given. Some results related to the hypercontractivity and the ultraboundedness of the semigroup are also established.
\end{abstract}

Keywords Vector-valued elliptic operators $\cdot$ Unbounded coefficients $\cdot$ Semigroups of bounded operators $\cdot$ Improving summability properties

Mathematics Subject Classification 35J47 · 35K45 · 47D06

The authors are members of G.N.A.M.P.A. of the Italian Istituto Nazionale di Alta Matematica (INdAM) and have been partially supported by the PRIN 2015 MIUR project 2015233N54.

Luciana Angiuli

luciana.angiuli@unisalento.it

Luca Lorenzi

luca.lorenzi@unipr.it

Elisabetta M. Mangino

elisabetta.mangino@unisalento.it

Abdelaziz Rhandi

arhandi@unisa.it

1 Dipartimento di Matematica e Fisica "Ennio De Giorgi”, Università del Salento, Via per Arnesano, I-73100 Lecce, Italy

2 Dipartimento di Scienze Matematiche, Fisiche e Informatiche, Plesso di Matematica, Università di Parma, Parco Area delle Scienze 53/A, I-43124 Parma, Italy

3 Dipartimento di Matematica, Università degli Studi di Salerno, Via Giovanni Paolo II 132, I- 84084 Fisciano (SA), Italy 


\section{Introduction}

Systems of elliptic equations with unbounded coefficients appear naturally in several settings, e.g., in the analysis of Navier-Stokes equations and backward-forward stochastic differential equations in connection with Nash equilibria in the theory of games, see e.g., $[1,20,23,24]$ and also $[10,11,21]$ for other relevant applications.

In this paper, we consider vector-valued elliptic operators with unbounded coefficients acting on smooth functions $f: \mathbb{R}^{d} \rightarrow \mathbb{R}^{m}(m \geq 2)$ as follows:

$$
\mathcal{A} \boldsymbol{f}=\operatorname{div}(Q \nabla \boldsymbol{f})+\sum_{i=1}^{d} B^{i} D_{i} \boldsymbol{f}-V \boldsymbol{f},
$$

where $Q, B^{i}(i=1, \ldots, d)$ and $V$ are matrix-valued functions, $Q$ is locally uniformly elliptic on $\mathbb{R}^{d}$ and $\left(\operatorname{div}(Q \nabla \boldsymbol{f})_{i}=\operatorname{div}\left(Q \nabla f_{i}\right)\right.$ for any $i=1, \ldots, m$.

Under suitable quantitative assumptions on the coefficients of the operator, we prove that the realization of $\mathcal{A}$ in $L^{p}\left(\mathbb{R}^{d} ; \mathbb{R}^{m}\right)$, with domain

$$
D_{p}=\left\{\boldsymbol{u} \in L^{p}\left(\mathbb{R}^{d} ; \mathbb{R}^{m}\right) \cap W_{\text {loc }}^{2, p}\left(\mathbb{R}^{d} ; \mathbb{R}^{m}\right): \operatorname{div}(Q \nabla \boldsymbol{u}), V \boldsymbol{u} \in L^{p}\left(\mathbb{R}^{d} ; \mathbb{R}^{m}\right)\right\},
$$

generates an analytic $C_{0}$-semigroup $\boldsymbol{T}_{p}(t)$. Refining gradually our main assumptions, we also prove the consistency of the semigroups $\boldsymbol{T}_{p}(t)=: \boldsymbol{T}(t)$ for $p \geq p_{0}$ and a suitable $p_{0}>1$ and for $p \in(1, \infty)$. We then show that each operator $\boldsymbol{T}(t)$ maps $L^{p}\left(\mathbb{R}^{d} ; \mathbb{R}^{m}\right)$ into $L^{q}\left(\mathbb{R}^{d} ; \mathbb{R}^{m}\right)$ for $p<q \leq \infty$.

To the best of our knowledge, this is the first paper aimed at providing a precise characterization of the domain of the infinitesimal generator of the associated semigroup, when also the diffusion coefficients of the operator $\mathcal{A}$ are possibly unbounded and the operator is coupled up to the first order. Indeed, the description of the domain of the generator of operator $\mathcal{A}$ in $L^{p}\left(\mathbb{R}^{d} ; \mathbb{R}^{m}\right)$ has been provided only in the papers $[22,25,28]$, but there the coefficients of the diffusion part are bounded, and in [7,8] where there is no coupling in the first-order term.

The literature on systems of elliptic operators with unbounded coefficients concerns mainly the analysis in spaces of bounded and continuous functions and the study of the so-called invariant measures (see, e.g., $[1-3,6,15]$ ). On the other hand, the $L^{p}$-theory is not well developed and the literature concerns essentially weakly coupled elliptic operators (i.e., the coupling between the equations is through a potential term), whose diffusion coefficients are assumed to be uniformly elliptic and bounded.

The first result in this direction is [22], whose assumptions allow the diagonal drift term to grow like $|x| \log (1+|x|)$ and the potential term like $\log (1+|x|)$ as $|x| \rightarrow \infty$, and the generation result is proved via a Dore-Venni-type theorem on sums of noncommuting operators, due to Monniaux and Prüss [32]. Note that this latter perturbation theorem requires information on the bounded imaginary powers of the realization in $L^{p}\left(\mathbb{R}^{d} ; \mathbb{R}^{m}\right)$ of the principal part of the operator $\mathcal{A}$. Unfortunately, such information for elliptic operators with unbounded coefficients is not available yet. The technique in [22] has been recently used in [25] to prove generation results in $L^{p}$-spaces for vector-valued Schrödinger operators of the form $\mathcal{A} \boldsymbol{u}=\operatorname{div}(Q \nabla \boldsymbol{u})-V \boldsymbol{u}$. In that paper, the entries of the potential $V$ of operator $\mathcal{A}$ are locally Lipschitz continuous on $\mathbb{R}^{d}$, satisfy the conditions $\langle V(x) \xi, \xi\rangle \geq|\xi|^{2}$, for every $x \in \mathbb{R}^{d}, \xi \in \mathbb{R}^{m}$, and $\left|D_{j} V(-V)^{-\alpha}\right| \in L^{\infty}\left(\mathbb{R}^{d}\right)$ for some $\alpha \in[0,1 / 2)$. The last assumption allows for potentials $V$ whose entries grow more than linearly at infinity. For instance, the potential $V(x)=\left(1+|x|^{r}\right) V_{0}$, for every $x \in \mathbb{R}^{d}$, 
where $V_{0}$ is an antisymmetric constant matrix and $r \in[1,2)$, is allowed, but no exponential growth rate at infinity is allowed.

Under slightly different hypotheses on the potential $V$ (pointwise accretivity and local boundedness), generation results for the operator $\mathcal{A}$ as above are proved in [26], but, differently from [25], only a weak characterization of the domain is provided (in fact, the generation result is proved in the maximal domain of the realization of the operator $\mathcal{A}$ in $\left.L^{p}\left(\mathbb{R}^{d} ; \mathbb{R}^{m}\right)\right)$.

A more general class of potentials, whose diagonal entries are polynomials of type $|x|^{\alpha}$ or even $|x|^{r} \log (1+|x|)$ as well as $e^{|x|}$, for $\alpha, r \geq 1$, is considered in [28] where the operator $\mathcal{A}$ is perturbed by the potential $v I$ where the function $v \in W_{\text {loc }}^{1, \infty}\left(\mathbb{R}^{d}\right)$ satisfies the condition $|\nabla v| \leq c v$ for some positive constant $c$. A perturbation theorem (due to Okazawa [33] and used in [28]) works for a more general diagonal perturbation of $V$ in the $L^{2}$-setting (see [7]), allowing for different growth rates in the diagonal entries of the potential matrix. In [7] the operator $\mathcal{A}$ is also perturbed by a diagonal first-order term that can grow at most linearly at infinity.

Assuming that the diffusion coefficients are bounded, our assumptions (see Hypotheses $3.1)$ cover the cases considered in $[22,25,26,28]$, where roughly speaking the coefficients may grow at most polynomially, allowing also for some exponential growth. We point out that, already in the case of Schrödinger vector-valued operators, our hypotheses allow for the entries of $V$ to grow at infinity as $e^{|x|^{\beta}}$, for every $\beta>0$, improving the growth-rate considered in [28] (see Example 5.1).

To our knowledge, the only other paper where systems of elliptic equations with unbounded diffusion coefficients are considered is [9]. In that paper, the semigroup constructed in the spaces of bounded and continuous functions is extrapolated to the $L^{p}$-scale, but the technique adopted does not provide any information on the domain of the infinitesimal generator of the semigroups. Moreover, the assumptions therein considered either impose a sign on the drift term and require that the quadratic form associated with the matrix-valued function $-2 V-\sum_{i} D_{i} B^{i}$ is bounded from above, or force the matrices $B^{i}$ to be bounded when the diffusion coefficients are themselves bounded. Such restrictions are not needed under our set of assumptions.

The present paper is organized as follows. In Sect. 2, we provide conditions that ensure that $C_{c}^{\infty}\left(\mathbb{R}^{d} ; \mathbb{R}^{m}\right)$ is a core for the maximal realization of operator $\mathcal{A}$ in $L^{p}\left(\mathbb{R}^{d} ; \mathbb{R}^{m}\right)$, when $\mathcal{A}$ is a vector-valued elliptic operator with a diagonal drift. Section 3 is the main body of the paper. Here, we introduce and comment the main assumptions and prove the generation result (see Theorem 3.3 and Proposition 3.9). Next, in Sect. 4 we first associate a semigroup in $C_{b}\left(\mathbb{R}^{d} ; \mathbb{R}^{m}\right)$ to the operator $\mathcal{A}$ and use this result to prove the consistency of the semigroups $\boldsymbol{T}_{p}(t)$ and summability improving results. Examples of classes of operators to which our results apply are provided in Sect. 5. Finally, some technicalities are postponed to appendix for an easier reading of the paper.

Notation. Let $d, m \in \mathbb{N}$ and let $\mathbb{K}=\mathbb{R}$ or $\mathbb{K}=\mathbb{C}$. We denote by $\langle\cdot, \cdot\rangle$ and by $|\cdot|$, respectively, the Euclidean inner product and the norm in $\mathbb{K}^{m}$. Vector-valued functions are displayed in bold style. Given a function $\boldsymbol{u}: \Omega \subseteq \mathbb{R}^{d} \rightarrow \mathbb{K}^{m}$, we denote by $u_{k}$ its $k$-th component. For every $p \in[1, \infty), L^{p}\left(\mathbb{R}^{d}, \mathbb{K}^{m}\right)$ denotes the classical vector-valued Lebesgue space endowed with the norm $\|\boldsymbol{f}\|_{p}=\left(\int_{\mathbb{R}^{d}}|\boldsymbol{f}(x)|^{p} d x\right)^{1 / p}$. The canonical pairing between $L^{p}\left(\mathbb{R}^{d}, \mathbb{K}^{m}\right)$ and $L^{p^{\prime}}\left(\mathbb{R}^{d}, \mathbb{K}^{m}\right)\left(p^{\prime}\right.$ being the index conjugate to $\left.p\right)$, i.e., the integral over $\mathbb{R}^{d}$ of the function $x \mapsto\langle\boldsymbol{u}(x), \boldsymbol{v}(x)\rangle$ when $\boldsymbol{u} \in L^{p}\left(\mathbb{R}^{d}, \mathbb{K}^{m}\right)$ and $\boldsymbol{v} \in L^{p^{\prime}}\left(\mathbb{R}^{d}, \mathbb{K}^{m}\right)$, is denoted by $\langle\boldsymbol{u}, \boldsymbol{v}\rangle_{p, p^{\prime}}$. For $k \in \mathbb{N}, W^{k, p}\left(\mathbb{R}^{d}, \mathbb{K}^{m}\right)$ is the classical vector-valued Sobolev space, i.e., the space of all functions $\boldsymbol{u} \in L^{p}\left(\mathbb{R}^{d}, \mathbb{K}^{m}\right)$ whose components have distributional derivatives up to the order $k$, which belong to $L^{p}\left(\mathbb{R}^{d}, \mathbb{K}\right)$. The norm of $W^{k, p}\left(\mathbb{R}^{d}, \mathbb{K}^{m}\right)$ is denoted by $\|\cdot\|_{k, p}$. 
Given a $d \times d$-matrix-valued function $Q$, we denote by $\mathfrak{q}(u, v)$ the function defined by $x \mapsto\langle Q(x) \nabla u(x), \nabla v(x)\rangle$ on smooth enough functions $u$ and $v$. We simply write $\mathfrak{q}(u)$ when $u=v$. Finally, given a vector-valued function $\boldsymbol{u}$ and $\varepsilon>0$, we denote by $|\boldsymbol{u}|_{\varepsilon}$ the scalar valued function $|\boldsymbol{u}|_{\varepsilon}=\left(|\boldsymbol{u}|^{2}+\varepsilon\right)^{1 / 2}$.

\section{Cores}

The aim of this section is to prove vector-valued versions of results about cores for elliptic operators with unbounded coefficients, in the line of those proved in [4]. Throughout the section, we will consider the elliptic operator $\mathcal{A}$ in (1.1) assuming that $B^{i}=b_{i} I$ for some functions $b_{i}: \mathbb{R}^{d} \rightarrow \mathbb{R}, i=1, \ldots, d$, and we set $\boldsymbol{b}=\left(b_{1}, \ldots, b_{d}\right)$.

In the following lemma we adapt some known results about scalar elliptic regularity to the vector-valued case.

Lemma 2.1 Suppose that $Q$ is symmetric and that $\langle Q(x) \xi, \xi\rangle>0$ for any $x \in \mathbb{R}^{d}$ and $\xi \in \mathbb{R}^{d} \backslash\{0\}$. Further, suppose that $q_{i j}, b_{i} \in C^{1}\left(\mathbb{R}^{d}\right)$ for $i, j=1, \ldots, d, v_{h k} \in L_{\text {loc }}^{\infty}\left(\mathbb{R}^{d}\right)$ for $h, k=1, \ldots, m$ and $\boldsymbol{u} \in L_{\mathrm{loc}}^{p}\left(\mathbb{R}^{d} ; \mathbb{R}^{m}\right), \boldsymbol{f} \in L_{\mathrm{loc}}^{p}\left(\mathbb{R}^{d} ; \mathbb{R}^{m}\right)$ for some $p \in(1, \infty)$ satisfy the variational formula

$$
\int_{\mathbb{R}^{d}} \boldsymbol{u} \mathcal{A} \boldsymbol{\varphi} d x=\int_{\mathbb{R}^{d}} \boldsymbol{f} \boldsymbol{\varphi} d x, \quad \boldsymbol{\varphi} \in C_{c}^{\infty}\left(\mathbb{R}^{d} ; \mathbb{R}^{m}\right) .
$$

Then, $\boldsymbol{u} \in W_{\text {loc }}^{2, p}\left(\mathbb{R}^{d} ; \mathbb{R}^{m}\right)$.

Proof Let $\boldsymbol{e}_{1}, \ldots, \boldsymbol{e}_{m}$ denote the canonical basis of $\mathbb{R}^{m}$. Writing (2.1) with $\boldsymbol{\varphi}=\varphi \mathbf{e}_{h}$ for some $\varphi \in C_{c}^{\infty}\left(\mathbb{R}^{d}\right)$ and $h \in\{1, \ldots, m\}$ we get

$$
\int_{\mathbb{R}^{d}}[\operatorname{div}(Q \nabla \varphi)+\langle\boldsymbol{b}, \nabla \varphi\rangle] u_{h} d x=\int_{\mathbb{R}^{d}}\left(f_{h}+\sum_{k=1}^{m} v_{k h} u_{k}\right) \varphi d x .
$$

By the arbitrariness of $h$ we get the assertion from the scalar case, by applying standard elliptic regularity (see, e.g., [27, Theorem D.1.4(iv)]).

Theorem 2.2 Let the hypotheses of Lemma 2.1 be satisfied. Further, assume that there exists a positive function $\psi \in C^{1}\left(\mathbb{R}^{d}\right)$ such that $\lim _{|x| \rightarrow \infty} \psi(x)=\infty$ and

$$
\begin{gathered}
\frac{\langle\boldsymbol{b}, \nabla \psi\rangle}{\psi \log \psi} \geq-C_{1} \text { and } \frac{\langle Q \nabla \psi, \nabla \psi\rangle}{(\psi \log \psi)^{2}} \leq C_{2}, \\
p^{-1}(\operatorname{div} \boldsymbol{b})|\xi|^{2}+\langle V \xi, \xi\rangle \geq 0
\end{gathered}
$$

in $\mathbb{R}^{d}$ for every $\xi \in \mathbb{R}^{m}$, some positive constants $C_{1}, C_{2}$ and some $p \in(1, \infty)$. Then, the operator $\left(\mathcal{A}, C_{c}^{\infty}\left(\mathbb{R}^{d} ; \mathbb{R}^{m}\right)\right)$ is closable on $L^{p}\left(\mathbb{R}^{d} ; \mathbb{R}^{m}\right)$ and its closure generates a strongly continuous semigroup.

Proof First, we observe that, thanks to $(2.4),\left(\mathcal{A}, C_{c}^{\infty}\left(\mathbb{R}^{d} ; \mathbb{R}^{m}\right)\right)$ is dissipative. Hence, by $\left[16\right.$, Proposition 3.14] we deduce that $\left(\mathcal{A}, C_{c}^{\infty}\left(\mathbb{R}^{d} ; \mathbb{R}^{m}\right)\right)$ is closable on $L^{p}\left(\mathbb{R}^{d} ; \mathbb{R}^{m}\right)$. Then, 
we have only to show that $(\lambda I-\mathcal{A})\left(C_{c}^{\infty}\left(\mathbb{R}^{d} ; \mathbb{R}^{m}\right)\right)$ is dense in $L^{p}\left(\mathbb{R}^{d} ; \mathbb{R}^{m}\right)$ for some $\lambda>0$. Since $\psi(x)$ tends to $\infty$ as $|x| \rightarrow \infty$, without loss of generality we can assume that $\psi(x) \geq 1$ for every $x \in \mathbb{R}^{d}$.

Fix $\lambda>0$ and let $\boldsymbol{u} \in L^{p^{\prime}}\left(\mathbb{R}^{d} ; \mathbb{R}^{m}\right)$ be such that $\langle\lambda \boldsymbol{\varphi}-\mathcal{A} \boldsymbol{\varphi}, \boldsymbol{u}\rangle_{p, p^{\prime}}=0$ or, equivalently, $\langle\mathcal{A} \boldsymbol{\varphi}, \boldsymbol{u}\rangle_{p, p^{\prime}}=\lambda\langle\boldsymbol{\varphi}, \boldsymbol{u}\rangle_{p, p^{\prime}}$ for every $\boldsymbol{\varphi} \in C_{c}^{\infty}\left(\mathbb{R}^{d} ; \mathbb{R}^{m}\right)$. We claim that $\boldsymbol{u} \in W_{\text {loc }}^{2, r}\left(\mathbb{R}^{d} ; \mathbb{R}^{m}\right)$ for some $r>d$ so that, in particular, $\boldsymbol{u} \in C^{1}\left(\mathbb{R}^{d} ; \mathbb{R}^{m}\right)$. For this purpose, we observe that, by Lemma $2.1, \boldsymbol{u} \in W_{\text {loc }}^{2, p^{\prime}}\left(\mathbb{R}^{d} ; \mathbb{R}^{m}\right)$. If $p^{\prime}>d$, then we are done. Otherwise, we apply the Sobolev embedding theorem, to infer that $\boldsymbol{u} \in L_{\text {loc }}^{q_{1}}\left(\mathbb{R}^{d} ; \mathbb{R}^{m}\right)$ for some $q_{1}>p^{\prime}$ and, consequently, by Lemma $2.1, \boldsymbol{u} \in W_{\text {loc }}^{2, q_{1}}\left(\mathbb{R}^{d} ; \mathbb{R}^{m}\right)$. If $q_{1}>d$, then we are done; otherwise, we iterate the procedure and in a finite number of steps we get the claim by a bootstrap argument.

Summing up, we have shown that

$$
\lambda \int_{\mathbb{R}^{d}}\langle\boldsymbol{\varphi}, \boldsymbol{u}\rangle d x=\int_{\mathbb{R}^{d}}\left(\sum_{h=1}^{m} \operatorname{div}\left(Q \nabla u_{h}\right) \varphi_{h}-\sum_{i=1}^{d} b_{i}\left\langle D_{i} \boldsymbol{u}, \boldsymbol{\varphi}\right\rangle-\left\langle\left(V^{*}+\operatorname{div} \boldsymbol{b}\right) \boldsymbol{u}, \boldsymbol{\varphi}\right\rangle\right) d x
$$

for every $\boldsymbol{\varphi} \in C_{c}^{\infty}\left(\mathbb{R}^{d} ; \mathbb{R}^{m}\right)$. Equality (2.5) extends by density to every function $\boldsymbol{\varphi} \in L^{p}\left(\mathbb{R}^{d} ; \mathbb{R}^{m}\right)$ with compact support.

Fix a smooth decreasing function $\zeta:[0, \infty) \rightarrow[0,1]$ such that $\zeta(s)=1$ if $s \in[0,1]$ and $\zeta(s)=0$ if $s \geq 2$, and set $\zeta_{n}=\zeta\left(n^{-1} \log \psi\right)$. Clearly $\zeta_{n} \in C_{c}^{\infty}\left(\mathbb{R}^{d}\right)$ and $\lim _{n \rightarrow \infty} \zeta_{n}(x)=1$ for every $x \in \mathbb{R}^{d}$. Note that the function $\boldsymbol{\varphi}_{n}:=\zeta_{n}^{2} \boldsymbol{u}|\boldsymbol{u}|_{\varepsilon}^{p^{p^{\prime}-2}}=\zeta_{n}^{2} \boldsymbol{u}\left(|\boldsymbol{u}|^{2}+\varepsilon\right)^{\left(p^{\prime}-2\right) / 2}$ belongs to $W^{1, p}\left(\mathbb{R}^{d} ; \mathbb{R}^{m}\right)\left(\right.$ since $\left.\boldsymbol{u} \in C^{1}\left(\mathbb{R}^{d} ; \mathbb{R}^{m}\right)\right)$ and has compact support. Hence, writing (2.5) with $\boldsymbol{\varphi}=\boldsymbol{\varphi}_{n}$ and integrating by parts the second-order term, we get

$$
\begin{aligned}
\lambda \int_{\mathbb{R}^{d}}|\boldsymbol{u}|^{2}|\boldsymbol{u}|_{\varepsilon}^{p^{\prime}-2} \zeta_{n}^{2} d x= & -\sum_{i=1}^{m} \int_{\mathbb{R}^{d}} \mathfrak{q}\left(\zeta_{n}^{2} u_{i}|\boldsymbol{u}|_{\varepsilon}^{p^{\prime}-2}, u_{i}\right) d x \\
& -\int_{\mathbb{R}^{d}}\langle(V+\operatorname{div} b) \boldsymbol{u}, \boldsymbol{u}\rangle \zeta_{n}^{2}|\boldsymbol{u}|_{\varepsilon}^{p^{\prime}-2} d x \\
& -\int_{\mathbb{R}^{d}} \zeta_{n}^{2} \sum_{i=1}^{d} \sum_{j=1}^{m} b_{i} D_{i} u_{j}\left(u_{j}|\boldsymbol{u}|_{\varepsilon}^{p^{\prime}-2}\right) d x \\
= & : \mathscr{I}_{1}-\int_{\mathbb{R}^{d}}\langle(V+\operatorname{div} \boldsymbol{b}) \boldsymbol{u}, \boldsymbol{u}\rangle \zeta_{n}^{2}|\boldsymbol{u}|_{\varepsilon}^{p^{\prime}-2} d x-\mathscr{I}_{2},
\end{aligned}
$$

where we recall that $\mathfrak{q}(f, g)=\langle Q \nabla f, \nabla g\rangle$ on smooth enough functions $f$ and $g$. Taking into account that $D_{i}|\boldsymbol{u}|_{\varepsilon}=|\boldsymbol{u}|_{\varepsilon}^{-1}\left\langle D_{i} \boldsymbol{u}, \boldsymbol{u}\right\rangle$ for every $i=1, \ldots, d$, it is easy to check that

$$
\begin{aligned}
\mathscr{I}_{1}= & -\sum_{i=1}^{m} \int_{\mathbb{R}^{d}} \mathfrak{q}\left(u_{i}\right) \zeta_{n}^{2}|\boldsymbol{u}|_{\varepsilon}^{p^{\prime}-2} d x-\int_{\mathbb{R}^{d}} \mathfrak{q}\left(\zeta_{n}^{2},|\boldsymbol{u}|_{\varepsilon}\right)|\boldsymbol{u}|_{\varepsilon}^{p^{\prime}-1} d x \\
& -\left(p^{\prime}-2\right) \int_{\mathbb{R}^{d}} \mathfrak{q}\left(|\boldsymbol{u}|_{\varepsilon}\right) \zeta_{n}^{2}|\boldsymbol{u}|_{\varepsilon}^{p^{\prime}-2} d x .
\end{aligned}
$$

Here, we remind that $\mathfrak{q}(f)=\mathfrak{q}(f, f)$. Moreover, applying Hölder's inequality we can estimate

$$
\left.\left.\left|\int_{\mathbb{R}^{d}} \mathfrak{q}\left(\zeta_{n}^{2},|\boldsymbol{u}|_{\varepsilon}\right)\right| \boldsymbol{u}\right|_{\varepsilon} ^{p^{\prime}-1} d x\left|\leq \delta \int_{\mathbb{R}^{d}} \mathfrak{q}\left(|\boldsymbol{u}|_{\varepsilon}\right)\right| \boldsymbol{u}\right|_{\varepsilon} ^{p^{\prime}-2} \zeta_{n}^{2} d x+\frac{1}{\delta} \int_{\mathbb{R}^{d}} \mathfrak{q}\left(\zeta_{n}\right)|\boldsymbol{u}|_{\varepsilon}^{p^{\prime}} d x
$$


for every $\delta>0$. Finally, observing that $\sum_{j=1}^{m} D_{i} u_{j}\left(u_{j}|\boldsymbol{u}|_{\varepsilon}^{p^{\prime}-2}\right)=p^{\prime-1} D_{i}|\boldsymbol{u}|_{\varepsilon}^{p^{\prime}} \quad$ and $|\boldsymbol{u}|_{\varepsilon}^{p^{\prime}}=|\boldsymbol{u}|^{2}|\boldsymbol{u}|_{\varepsilon}^{p^{\prime}-2}+\varepsilon|\boldsymbol{u}|_{\varepsilon}^{p^{\prime}-2}$, integrating by parts we obtain

$$
\begin{aligned}
\mathscr{I}_{2}= & -\frac{1}{p^{\prime}} \int_{\mathbb{R}^{d}}(\operatorname{div} \boldsymbol{b})|\boldsymbol{u}|_{\varepsilon}^{p^{\prime}} \zeta_{n}^{2} d x-\frac{1}{p^{\prime}} \int_{\mathbb{R}^{d}}\left\langle\boldsymbol{b}, \nabla \zeta_{n}^{2}\right\rangle|\boldsymbol{u}|_{\varepsilon}^{p^{\prime}} d x \\
= & -\frac{1}{p^{\prime}} \int_{\mathbb{R}^{d}}(\operatorname{div} \boldsymbol{b})|\boldsymbol{u}|^{2}|\boldsymbol{u}|_{\varepsilon}^{p^{\prime}-2} \zeta_{n}^{2} d x-\frac{\varepsilon}{p^{\prime}} \int_{\mathbb{R}^{d}}(\operatorname{div} \boldsymbol{b})|\boldsymbol{u}|_{\varepsilon}^{p^{\prime}-2} \zeta_{n}^{2} d x \\
& -\frac{1}{p^{\prime}} \int_{\mathbb{R}^{d}}\left\langle\boldsymbol{b}, \nabla \zeta_{n}^{2}\right\rangle|\boldsymbol{u}|_{\varepsilon}^{p^{\prime}} d x .
\end{aligned}
$$

Replacing (2.7) and (2.9) in formula (2.6), we obtain

$$
\begin{aligned}
\lambda \int_{\mathbb{R}^{d}}|\boldsymbol{u}|^{2}|\boldsymbol{u}|_{\varepsilon}^{p^{\prime}-2} \zeta_{n}^{2} d x= & -\int_{\mathbb{R}^{d}} \sum_{i=1}^{m} \mathfrak{q}\left(u_{i}\right) \zeta_{n}^{2}|\boldsymbol{u}|_{\varepsilon}^{p^{\prime}-2} d x-\int_{\mathbb{R}^{d}} \mathfrak{q}\left(\zeta_{n}^{2},|\boldsymbol{u}|_{\varepsilon}\right)|\boldsymbol{u}|_{\varepsilon}^{p^{\prime}-1} d x \\
& -\left(p^{\prime}-2\right) \int_{\mathbb{R}^{d}} \mathfrak{q}\left(|\boldsymbol{u}|_{\varepsilon}\right) \zeta_{n}^{2}|\boldsymbol{u}|_{\varepsilon}^{p^{\prime}-2} d x \\
& -\int_{\mathbb{R}^{d}}\left\langle\left(V+p^{-1} \operatorname{div} \boldsymbol{b}\right) \boldsymbol{u}, \boldsymbol{u}\right\rangle \zeta_{n}^{2}|\boldsymbol{u}|_{\varepsilon}^{p^{\prime}-2} d x \\
& +\frac{\varepsilon}{p^{\prime}} \int_{\mathbb{R}^{d}}(\operatorname{div} \boldsymbol{b})|\boldsymbol{u}|_{\varepsilon}^{p^{\prime}-2} \zeta_{n}^{2} d x+\frac{1}{p^{\prime}} \int_{\mathbb{R}^{d}}\left\langle\boldsymbol{b}, \nabla \zeta_{n}^{2}\right\rangle|\boldsymbol{u}|_{\varepsilon}^{p^{\prime}} d x .
\end{aligned}
$$

We now distinguish the cases $p<2$ and $p \geq 2$. In the first, case, $p^{\prime}-2>0$ so that using (2.8), with $\delta<p^{\prime}-2$, in (2.10), disregarding the first and the fourth terms in the righthand side of (2.10), since they are nonpositive (take (2.4) into account), we get

$$
\begin{aligned}
& \lambda \int_{\mathbb{R}^{d}}|\boldsymbol{u}|^{2}|\boldsymbol{u}|_{\varepsilon}^{p^{\prime}-2} \zeta_{n}^{2} d x \\
& \leq \frac{1}{\delta} \int_{\mathbb{R}^{d}} \mathfrak{q}\left(\zeta_{n}\right)|\boldsymbol{u}|_{\varepsilon}^{p^{\prime}} d x+\frac{\varepsilon}{p^{\prime}} \int_{\mathbb{R}^{d}}(\operatorname{div} \boldsymbol{b}) \zeta_{n}^{2}|\boldsymbol{u}|_{\varepsilon}^{p^{\prime}-2} d x+\frac{1}{p^{\prime}} \int_{\mathbb{R}^{d}}\left\langle\boldsymbol{b}, \nabla \zeta_{n}^{2}\right\rangle|\boldsymbol{u}|_{\varepsilon}^{p^{\prime}} d x \\
& \leq \frac{1}{\delta n^{2}} \int_{\mathbb{R}^{d}} \frac{\mathfrak{q}(\psi)}{\psi^{2}}\left[\zeta^{\prime}\left(n^{-1} \log \psi\right)\right]^{2}|\boldsymbol{u}|_{\varepsilon}^{p^{\prime}} d x+\frac{\varepsilon}{p^{\prime}} \int_{\mathbb{R}^{d}}(\operatorname{div} \boldsymbol{b}) \zeta_{n}^{2}|\boldsymbol{u}|_{\varepsilon}^{p^{\prime}-2} d x \\
& \quad+\frac{2}{p^{\prime} n} \int_{\mathbb{R}^{d}} \frac{\langle\boldsymbol{b}, \nabla \psi\rangle}{\psi} \zeta^{\prime}\left(n^{-1} \log \psi\right) \zeta_{n}|\boldsymbol{u}|_{\varepsilon}^{p^{\prime}} d x .
\end{aligned}
$$

If $p \geq 2$, then $p^{\prime}-2$ is nonpositive and we write

$$
\begin{aligned}
\int_{\mathbb{R}^{d}} \mathfrak{q}\left(|\boldsymbol{u}|_{\varepsilon}\right) \zeta_{n}^{2}|\boldsymbol{u}|_{\varepsilon}^{p^{\prime}-2} d x & =\frac{1}{4} \int_{\mathbb{R}^{d}} \mathfrak{q}\left(|\boldsymbol{u}|_{\varepsilon}^{2}\right) \zeta_{n}^{2}|\boldsymbol{u}|_{\varepsilon}^{p^{\prime}-4} d x \\
& =\int_{\mathbb{R}^{d}} \sum_{i, j=1}^{d} q_{i j}\left\langle D_{i} \boldsymbol{u}, \boldsymbol{u}\right\rangle\left\langle D_{j} \boldsymbol{u}, \boldsymbol{u}\right\rangle \zeta_{n}^{2}|\boldsymbol{u}|_{\varepsilon}^{p^{\prime}-4} d x
\end{aligned}
$$

so that 


$$
\begin{aligned}
\left|p^{\prime}-2\right| \int_{\mathbb{R}^{d}} \mathfrak{q}\left(|\boldsymbol{u}|_{\varepsilon}\right) \zeta_{n}^{2}|\boldsymbol{u}|_{\varepsilon}^{p^{\prime}-2} d x & \leq\left(2-p^{\prime}\right) \int_{\mathbb{R}^{d}} \sum_{h, k=1}^{m}\left|\mathfrak{q}\left(u_{h}, u_{k}\right)\right|\left|u_{h}\right|\left|u_{k}\right| \zeta_{n}^{2}|\boldsymbol{u}|_{\varepsilon}^{p^{\prime}-4} d x \\
& \leq\left(2-p^{\prime}\right) \int_{\mathbb{R}^{d}}\left(\sum_{h=1}^{m}\left|Q^{1 / 2} \nabla u_{h}\right|\left|u_{h}\right|\right)^{2} \zeta_{n}^{2}|\boldsymbol{u}|_{\varepsilon}^{p^{\prime}-4} d x \\
& \leq\left(2-p^{\prime}\right) \sum_{h=1}^{m} \int_{\mathbb{R}^{d}} \mathfrak{q}\left(u_{h}\right) \zeta_{n}^{2}|\boldsymbol{u}|_{\varepsilon}^{p^{\prime}-2} d x .
\end{aligned}
$$

Therefore,

$$
\begin{aligned}
& -\int_{\mathbb{R}^{d}} \sum_{i=1}^{m} \mathfrak{q}\left(u_{i}\right) \zeta_{n}^{2}|\boldsymbol{u}|_{\varepsilon}^{p^{\prime}-2} d x-\left(p^{\prime}-2\right) \int_{\mathbb{R}^{d}} \mathfrak{q}\left(|\boldsymbol{u}|_{\varepsilon}\right) \zeta_{n}^{2}|\boldsymbol{u}|_{\varepsilon}^{p^{\prime}-2} d x \\
& \quad \leq\left(1-p^{\prime}\right) \int_{\mathbb{R}^{d}} \sum_{i=1}^{m} \mathfrak{q}\left(u_{i}\right) \zeta_{n}^{2}|\boldsymbol{u}|_{\varepsilon}^{p^{\prime}-2} d x .
\end{aligned}
$$

Now, we take $\delta<p^{\prime}-1$ and, proceeding as in the case $p^{\prime}<2$, we still obtain (2.11).

Note that the second integral in the last side of (2.11) converges to 0 as $\varepsilon \rightarrow 0$. Indeed, for each $\varepsilon \in(0,1)$ we can estimate

$$
\varepsilon \int_{\mathbb{R}^{d}}(\operatorname{div} \boldsymbol{b}) \zeta_{n}^{2}|\boldsymbol{u}|_{\varepsilon}^{p^{\prime}-2} d x \leq \varepsilon^{\frac{p^{\prime} \wedge 2}{2}} \int_{\mathbb{R}^{d}}|\operatorname{div} \boldsymbol{b}||\boldsymbol{u}|_{1}^{\left(p^{\prime}-2\right)^{+}} \zeta_{n}^{2} d x
$$

which vanishes as $\varepsilon \rightarrow 0^{+}$since the function $\zeta_{n}$ is compactly supported in $\mathbb{R}^{d}$ and the functions $\operatorname{div} \boldsymbol{b},|\boldsymbol{u}|_{1}$ are, respectively, locally integrable and locally bounded on $\mathbb{R}^{d}$. Here, $\left(p^{\prime}-2\right)^{+}$denotes the positive part of $p^{\prime}-2$. Hence, letting $\varepsilon \rightarrow 0$ and using the dominated convergence theorem, we deduce that

$$
\begin{aligned}
\lambda \int_{\mathbb{R}^{d}}|\boldsymbol{u}|^{p^{\prime}} \zeta_{n}^{2} d x \leq & \frac{1}{\delta n^{2}} \int_{\mathbb{R}^{d}} \frac{\mathfrak{q}(\psi)}{\psi^{2}}\left[\zeta^{\prime}\left(n^{-1} \log \psi\right)\right]^{2}|\boldsymbol{u}|^{p^{\prime}} d x \\
& +\frac{2}{p^{\prime} n} \int_{\mathbb{R}^{d}} \frac{\langle\boldsymbol{b}, \nabla \psi\rangle}{\psi} \zeta^{\prime}\left(n^{-1} \log \psi\right) \zeta_{n}|\boldsymbol{u}|^{p^{\prime}} d x .
\end{aligned}
$$

Since, for every $n \in \mathbb{N}, \zeta^{\prime}\left(n^{-1} \log \psi(x)\right) \neq 0$ only if $1 \leq n^{-1} \log \psi(x) \leq 2$, taking (2.3) into account we can estimate

$$
\left.\left.\left|\frac{1}{\delta n^{2}} \frac{\mathfrak{q}(\psi)}{\psi^{2}}\left[\zeta^{\prime}\left(n^{-1} \log \psi\right)\right]^{2}\right| \boldsymbol{u}\right|^{p^{\prime}}\left|\leq \frac{4 \mathfrak{q}(\psi)}{\delta \psi^{2} \log ^{2} \psi}\left\|\zeta^{\prime}\right\|_{\infty}^{2}\right| \boldsymbol{u}\right|^{p^{\prime}} \leq 4 C_{2} \delta^{-1}\left\|\zeta^{\prime}\right\|_{\infty}^{2}|\boldsymbol{u}|^{p^{\prime}} .
$$

Moreover, since $\zeta^{\prime} \leq 0$ on $[0, \infty)$, it follows that

$$
\frac{1}{p^{\prime} n} \int_{\mathbb{R}^{d}} \frac{\langle\boldsymbol{b}, \nabla \psi\rangle}{\psi} \zeta^{\prime}\left(n^{-1} \log \psi\right) \zeta_{n}|\boldsymbol{u}|^{p^{\prime}} d x \leq \frac{2 C_{1}}{p^{\prime}}\left\|\zeta^{\prime}\right\|_{\infty} \int_{\mathbb{R}^{d}}|\boldsymbol{u}|^{p^{\prime}} d x .
$$

Hence, by dominated convergence we can let $n$ tend to $\infty$ in both sides of (2.12) and conclude that $\lambda\|\boldsymbol{u}\|_{p^{\prime}} \leq 0$, whence $\boldsymbol{u}=\mathbf{0}$.

Theorem 2.3 In addition to the hypotheses of Lemma 2.1, let us assume that condition (2.4) holds true and that there exists a positive function $\psi \in C^{1}\left(\mathbb{R}^{d}\right)$, which diverges to $\infty$ as $|x|$ tends to $\infty$, such that 


$$
\left|\frac{\langle\boldsymbol{b}, \nabla \psi\rangle}{\psi \log \psi}\right| \leq C_{1} \text { and } \frac{\langle Q \nabla \psi, \nabla \psi\rangle}{(\psi \log \psi)^{2}} \leq C_{2}
$$

for some constants $C_{1}, C_{2}>0$. Then, the realization $A_{p}$ of the operator $\mathcal{A}$ in $L^{p}\left(\mathbb{R}^{d}, \mathbb{R}^{m}\right)$, with domain $D_{p, \max }=\left\{\boldsymbol{u} \in L^{p}\left(\mathbb{R}^{d} ; \mathbb{R}^{m}\right) \cap W_{\text {loc }}^{2, p}\left(\mathbb{R}^{d} ; \mathbb{R}^{m}\right): \mathcal{A} \boldsymbol{u} \in L^{p}\left(\mathbb{R}^{d} ; \mathbb{R}^{m}\right)\right\}$, generates a contraction semigroup in $L^{p}\left(\mathbb{R}^{d} ; \mathbb{R}^{m}\right)$. Moreover, the space $C_{c}^{\infty}\left(\mathbb{R}^{d} ; \mathbb{R}^{m}\right)$ is a core for $\left(\mathcal{A}, D_{p, \text { max }}\right)$.

In the particular case when $\boldsymbol{b}$ identically vanishes and $\langle V(x) \xi, \xi\rangle \geq 0$ for every $x, \xi \in \mathbb{R}^{d}$, the previous semigroups exist for every $p \in(1, \infty)$ and are consistent.

Proof Let $(\overline{\mathcal{A}}, D)$ be the closure of $\left(\mathcal{A}, C_{c}^{\infty}\left(\mathbb{R}^{d} ; \mathbb{R}^{m}\right)\right)$ in $L^{p}\left(\mathbb{R}^{d} ; \mathbb{R}^{m}\right)$ and fix $\boldsymbol{u} \in D$. Then, there exists a sequence $\left(\boldsymbol{u}_{n}\right)$ in $C_{c}^{\infty}\left(\mathbb{R}^{d} ; \mathbb{R}^{m}\right)$ such that $\boldsymbol{u}_{n}$ and $\mathcal{A} \boldsymbol{u}_{n}$ converge, respectively to some function $\boldsymbol{u}$ and $\boldsymbol{g}$ in $L^{p}\left(\mathbb{R}^{d} ; \mathbb{R}^{m}\right)$, as $n$ tends to $\infty$. Hence, for every $\boldsymbol{\varphi} \in C_{c}^{\infty}\left(\mathbb{R}^{d} ; \mathbb{R}^{m}\right)$ it follows that

$$
\int_{\mathbb{R}^{d}}\left\langle\boldsymbol{u}, \mathcal{A}^{*} \boldsymbol{\varphi}\right\rangle d x=\int_{\mathbb{R}^{d}}\langle\boldsymbol{g}, \boldsymbol{\varphi}\rangle d x
$$

where $\mathcal{A}^{*}$ is the formal adjoint to the operator $\mathcal{A}$, which implies that $\overline{\mathcal{A}} \boldsymbol{u}=\boldsymbol{g}=\mathcal{A} \boldsymbol{u}$ distributionally. By Lemma 2.1, we deduce that $\boldsymbol{u} \in D_{p, \max }$.

Let us now prove that $\lambda I-\mathcal{A}$ is injective on $D_{p, \max }$ for some $\lambda>0$. For this purpose, we fix $\boldsymbol{u} \in D_{p \text {,max }}$ such that $\lambda \boldsymbol{u}=\mathcal{A} \boldsymbol{u}$. Then, for every $\boldsymbol{\varphi} \in C_{c}^{\infty}\left(\mathbb{R}^{d} ; \mathbb{R}^{m}\right)$, it holds that $\left\langle\boldsymbol{u}, \lambda \boldsymbol{\varphi}-\mathcal{A}^{*} \boldsymbol{\varphi}\right\rangle_{p, p^{\prime}}=\langle\lambda \boldsymbol{u}-\mathcal{A} \boldsymbol{u}, \boldsymbol{\varphi}\rangle_{p, p^{\prime}}=0$. Since $C_{c}^{\infty}\left(\mathbb{R}^{d} ; \mathbb{R}^{m}\right)$ is a core for $\mathcal{A}^{*}$ in $L^{p^{\prime}}\left(\mathbb{R}^{d} ; \mathbb{R}^{m}\right)$, due to Theorem 2.2, we conclude that $\boldsymbol{u}=\mathbf{0}$.

Next, we fix a function $\boldsymbol{u} \in D_{p \text {, max }}$ and set $\boldsymbol{v}=\lambda \boldsymbol{u}-\mathcal{A} \boldsymbol{u}$. By Theorem 2.2, $\boldsymbol{v}=\lambda \boldsymbol{w}-\overline{\mathcal{A}} \boldsymbol{w}=\lambda \boldsymbol{w}-\mathcal{A} \boldsymbol{w}$ for some $\boldsymbol{w} \in D \subseteq D_{p, \max }$. By the injectivity of $\mathcal{A}$ on $D_{p, \max }$ we get that $u=w \in D$.

Finally, let us assume that $\boldsymbol{b}$ identically vanishes on $\mathbb{R}^{d}$. To prove that the semigroups generated by the operators $\boldsymbol{A}_{p}$ and $\boldsymbol{A}_{q}$ are consistent, one can take advantage of the Trotter product formula (see [16, Corollary III.5.8]) to write

$$
e^{t \boldsymbol{A}_{r}} \boldsymbol{f}=\lim _{n \rightarrow \infty}\left(e^{\frac{t}{n} \boldsymbol{A}_{r}^{0}} e^{-\frac{t}{n} \boldsymbol{V}_{r}}\right)^{n} \boldsymbol{f}, \quad \boldsymbol{f} \in L^{r}\left(\mathbb{R}^{d} ; \mathbb{R}^{m}\right),
$$

for every $t>0$ and $r \in\{p, q\}$ if $p, q \geq 2$, where $e^{-t V_{r}}$ is the strongly semigroup in $L^{r}\left(\mathbb{R}^{d} ; \mathbb{R}^{m}\right)$ generated by the multiplication operator $\boldsymbol{u} \mapsto-V \boldsymbol{u}$, with $D\left(\boldsymbol{V}_{r}\right)=\left\{\boldsymbol{u} \in L^{r}\left(\mathbb{R}^{d} ; \mathbb{R}^{m}\right): V \boldsymbol{u} \in L^{r}\left(\mathbb{R}^{d} ; \mathbb{R}^{m}\right)\right\}$ and $e^{t \boldsymbol{A}_{r}^{0} \boldsymbol{f}}=\left(e^{t A_{r}^{0}} f_{1}, \ldots, e^{t A_{r}^{0}} f_{m}\right)$ for every $t>0$ and $f \in L^{r}\left(\mathbb{R}^{d} ; \mathbb{R}^{m}\right)$, where $e^{t A_{r}^{0}}$ is the scalar semigroup generated by the operator $\operatorname{div}(Q \nabla)$. Both the semigroups $e^{t A_{r}^{0}}$ and $e^{-t V_{r}}$ are consistent on the $L^{p}$-scale. If $p, q \in(1,2]$, then we observe that the operator $\mathcal{A}^{*}$ adjoint to $\mathcal{A}$ satisfies the same assumptions as the operator $\mathcal{A}$. Therefore, for $r \in\{p, q\}$, the semigroup $e^{t \boldsymbol{A}_{r}}$ is the adjoint of the semigroup generated in $L^{r^{\prime}}\left(\mathbb{R}^{d} ; \mathbb{R}^{m}\right)$ by the closure of the operator $\left(\mathcal{A}^{*}, C_{c}^{\infty}\left(\mathbb{R}^{d} ; \mathbb{R}^{m}\right)\right)$. Denote by $\boldsymbol{T}_{r^{\prime}}(t)$ this semigroup. Then, for every $t>0, f \in L^{p}\left(\mathbb{R}^{d} ; \mathbb{R}^{m}\right) \cap L^{q}\left(\mathbb{R}^{d} ; \mathbb{R}^{m}\right)$ and $\boldsymbol{\varphi} \in C_{c}^{\infty}\left(\mathbb{R}^{d} ; \mathbb{R}^{m}\right)$, we can write

$$
\left\langle e^{t \boldsymbol{A}_{p}} \boldsymbol{f}, \boldsymbol{\varphi}\right\rangle_{p, p^{\prime}}=\left\langle\boldsymbol{f}, \boldsymbol{T}_{p^{\prime}}(t) \boldsymbol{\varphi}\right\rangle_{p, p^{\prime}}=\left\langle\boldsymbol{f}, \boldsymbol{T}_{q^{\prime}}(t) \boldsymbol{\varphi}\right\rangle_{q, q^{\prime}}=\left\langle e^{t \boldsymbol{A}_{q}} \boldsymbol{f}, \boldsymbol{\varphi}\right\rangle_{q, q^{\prime}}
$$

and the equality $e^{t \boldsymbol{A}_{p}} \boldsymbol{f}=e^{t \boldsymbol{A}_{q}} \boldsymbol{f}$ follows. Finally, if $p<2$ and $q>2$, then $e^{t \boldsymbol{A}_{p}} \boldsymbol{f}=e^{t \boldsymbol{A}_{2}} \boldsymbol{f}=e^{t \boldsymbol{A}}{ }_{q} \boldsymbol{f}$ for every $t>0$ and $f \in C_{c}^{\infty}\left(\mathbb{R}^{d} ; \mathbb{R}^{m}\right)$. Approximating $f \in L^{p}\left(\mathbb{R}^{d} ; \mathbb{R}^{m}\right) \cap L^{q}\left(\mathbb{R}^{d} ; \mathbb{R}^{m}\right)$ in 
$L^{p}\left(\mathbb{R}^{d} ; \mathbb{R}^{m}\right) \cap L^{q}\left(\mathbb{R}^{d} ; \mathbb{R}^{m}\right)$ with a sequence of functions of function $\left(\boldsymbol{f}_{n}\right) \subset C_{c}^{\infty}\left(\mathbb{R}^{d} ; \mathbb{R}^{m}\right)$, the equality $e^{t \boldsymbol{A}_{p}}=e^{t \boldsymbol{A}_{q}}$ on $L^{p}\left(\mathbb{R}^{d} ; \mathbb{R}^{m}\right) \cap L^{q}\left(\mathbb{R}^{d} ; \mathbb{R}^{m}\right)$ follows for every $t>0$ also in this case.

\section{The full operator $\mathcal{A}$}

In this section, we consider the elliptic operator $\mathcal{A}$ defined in (1.1) assuming that $p \in(1, \infty)$ and that the coefficients $Q=\left(q_{i j}\right), B^{i}=\left(B_{h k}^{i}\right), V=\left(v_{h k}\right)$ satisfy the following assumptions:

\section{Hypotheses 3.1}

(i) $Q$ is a real, symmetric matrix with entries in $C^{1}\left(\mathbb{R}^{d}\right)$. Moreover, $\langle Q(x) \xi, \xi\rangle>0$ for every $x \in \mathbb{R}^{d}$ and $\xi \in \mathbb{R}^{d} \backslash\{0\}$;

(ii) $B^{i}: \mathbb{R}^{d} \rightarrow \mathbb{R}^{m \times m}$ are symmetric matrix-valued functions, with coefficients of class $C^{1}$ over $\mathbb{R}^{d}$ for every $i=1, \ldots, d$;

(iii) $\quad V: \mathbb{R}^{d} \rightarrow \mathbb{R}^{m \times m}$ is a measurable matrix-valued function;

(iv) there exists a function $v \in C^{1}\left(\mathbb{R}^{d}\right)$ with positive infimum $c_{0}$ and positive constants $\kappa, c_{1}$ and $\theta<p$ such that

$$
\begin{aligned}
& \langle V(x) \xi, \xi\rangle \geq v(x)|\xi|^{2}, \quad|V(x) \xi| \leq c_{1} v(x)|\xi|, \\
& \sum_{h, k=1}^{m}\left|\sum_{i=1}^{d} B_{h k}^{i}(x) \eta_{i}^{k}\right| \leq \kappa \sqrt{v(x)} \sum_{k=1}^{m}\left\langle Q(x) \eta^{k}, \eta^{k}\right\rangle^{\frac{1}{2}}, \\
& \langle\operatorname{div} B(x) \xi, \xi\rangle \geq-\theta v(x)|\xi|^{2}
\end{aligned}
$$

for every $x \in \mathbb{R}^{d}, \xi \in \mathbb{R}^{m}, \eta^{k}=\left(\eta_{1}^{k}, \ldots, \eta_{d}^{k}\right) \in \mathbb{R}^{d}$ and $k=1, \ldots, m$, where we set $\operatorname{div} B(x)=\sum_{i=1}^{d} D_{i} B^{i}(x)$ for every $x \in \mathbb{R}^{d}, D_{i} B^{i}$ being the matrix whose entries are obtained differentiating with respect to the variable $x_{i}$ the corresponding entries of the matrix $B^{i}$;

(v) there exist positive constants $\gamma, C_{\gamma}$ and $c_{2}$ such that

$$
\begin{array}{ll}
\text { if } 1<p \leq 2: & \langle Q(x) \nabla v(x), \nabla v(x)\rangle^{\frac{1}{2}} \leq \gamma v(x)^{3 / 2}+C_{\gamma} \\
\text { if } p>2: & \left\{\begin{array}{l}
|Q(x)|^{\frac{1}{2}}|\nabla v(x)| \leq \gamma v(x)^{3 / 2}+C_{\gamma}, \\
|Q(x)| \leq c_{2} v(x), \\
\sup _{|x-y| \leq \rho(x)}|\nabla Q(y)| \leq c_{2} \mu(x) \rho(x)^{-1}
\end{array}\right.
\end{array}
$$

for every $x \in \mathbb{R}_{1}^{d}$, where $\mu(x)$ is the minimum eigenvalue of $Q(x)$ and $\rho(x)=|Q(x)|^{\frac{1}{2}} v(x)^{-\frac{1}{2}}$

(vi) there exists a positive function $\psi \in C^{1}\left(\mathbb{R}^{d}\right)$ such that $\lim _{|x| \rightarrow \infty} \psi(x)=\infty$ and $\langle Q \nabla \psi, \nabla \psi\rangle \leq C \psi^{2} \log ^{2} \psi$.

Remark 3.2 The above assumptions might seem somehow restrictive and only technical. Actually, elliptic operators with unbounded coefficients exhibit a bad behavior in the usual $L^{p}$-spaces with respect to the Lebesgue measure unless suitable (strong) conditions are 
assumed on their coefficients. An easy scalar one-dimensional counterexample shows that, even in the case when the diffusion coefficient of the operator $\mathcal{A}$ is constant and the drift grows slightly more than linearly at infinity, there exists no realization of the operator $\mathcal{A}$ in $L^{p}(\mathbb{R})$ which generates a strongly continuous or an analytic semigroup (see [34]). Similar pathological behaviors are exhibited in the vector-valued case (see, e.g., [22, Example 2.2], [25, Example 2.3], [9, Sect. 3]). So, in order to prove generation results, one has to require strong conditions on the growth of the drift term or, as an alternative, to assume the existence of a dominating potential term .

Our assumptions on the diffusion coefficients of operator $\mathcal{A}$ are inspired by those considered in [29] (see also [12,13]) where the scalar equation is studied. Hypotheses 3.1(iv), (vi) guarantee the sectoriality of the operator $-\mathcal{A}$, in the sense of [19], Definitions 1.5.8. On the other hand, inequality (3.1) and the oscillation condition in Hypothesis 3.1(v) are crucial to interpolate the term $\sum_{i=1}^{d} B_{i} D_{i} \boldsymbol{u}$ between $\operatorname{div}(Q \nabla \boldsymbol{u})$ and $V \boldsymbol{u}$.

For every $p \in(1, \infty)$, let $\boldsymbol{A}_{p}$ denote the realization of the operator $\mathcal{A}$ in $L^{p}\left(\mathbb{R}^{d} ; \mathbb{R}^{m}\right)$ with domain

$$
\begin{aligned}
D_{p} & =\left\{\boldsymbol{u} \in W_{\mathrm{loc}}^{2, p}\left(\mathbb{R}^{d} ; \mathbb{C}^{m}\right): \boldsymbol{u}, \mathcal{A}_{0} \boldsymbol{u}, V \boldsymbol{u} \in L^{p}\left(\mathbb{R}^{d} ; \mathbb{C}^{m}\right)\right\} \\
& =\left\{\boldsymbol{u} \in W_{\mathrm{loc}}^{2, p}\left(\mathbb{R}^{d} ; \mathbb{C}^{m}\right): \mathcal{A}_{0} \boldsymbol{u}, v \boldsymbol{u} \in L^{p}\left(\mathbb{R}^{d} ; \mathbb{C}^{m}\right)\right\}
\end{aligned}
$$

where $\mathcal{A}_{0} \boldsymbol{u}:=\operatorname{div}(Q \nabla \boldsymbol{u})$. On $D_{p}$ we consider the norm $\|\boldsymbol{u}\|_{D_{p}}=\left\|\mathcal{A}_{0} \boldsymbol{u}\right\|_{p}+\|v \boldsymbol{u}\|_{p}$, which is clearly equivalent to the norm $\boldsymbol{u} \mapsto\|\boldsymbol{u}\|_{p}+\left\|\mathcal{A}_{0} \boldsymbol{u}\right\|_{p}+\|V \boldsymbol{u}\|_{p}$ due to Hypothesis 3.1(iv) which implies that $\|v \boldsymbol{u}\|_{p} \leq\|V \boldsymbol{u}\|_{p} \leq c_{1}\|v \boldsymbol{u}\|_{p}$ and $\|\boldsymbol{u}\|_{p} \leq c_{0}^{-1}\|v \boldsymbol{u}\|_{p}$ for every $\boldsymbol{u} \in L^{p}\left(\mathbb{R}^{d} ; \mathbb{C}^{m}\right)$. Since $\mathcal{A}_{0}$ is a closed operator, $D_{p}$ endowed with the norm $\|\cdot\|_{D_{p}}$ is a Banach space.

We can now state the main generation result:

Theorem 3.3 Under Hypotheses 3.1, assume further the condition

$$
1-\frac{\theta}{p}-\frac{p-1}{p} \kappa m \gamma-\frac{1}{4(p-1)}\left[(3-p) \gamma+\frac{\kappa(2-p)}{p}(\sqrt{m}+m)\right]^{2}>0,
$$

if $p \in(1,2)$, and the condition

$$
1-\frac{\theta}{p}-\gamma\left(\frac{p-1}{p} \kappa m+\frac{\gamma}{4}(p-1)+\frac{p-2}{2 p} \kappa(\sqrt{m}+m)\right)-\frac{p-2}{4 p^{2}} \kappa^{2} m(m+p-2)>0,
$$

if $p \geq 2$. Then, the operator $\boldsymbol{A}_{p}$ generates an analytic contraction semigroup $\boldsymbol{T}_{p}(t)$ in $L^{p}\left(\mathbb{R}^{d} ; \mathbb{C}^{m}\right)$. Moreover, if the above assumptions are satisfied also for some $1<q \neq p$, then $\boldsymbol{T}_{p}(t) \boldsymbol{f}=\boldsymbol{T}_{q}(t) \boldsymbol{f}$ for all $\boldsymbol{f} \in L^{p}\left(\mathbb{R}^{d} ; \mathbb{C}^{m}\right) \cap L^{q}\left(\mathbb{R}^{d} ; \mathbb{C}^{m}\right)$. 


\section{Remark 3.4}

(i) We stress that, without assuming conditions like (3.2) and (3.3), the domain characterization in Theorem 3.3 could fail, as some scalar counterexamples show (see [31, Example 3.7]).

(ii) We also point out that, if Hypothesis 3.1(v) is satisfied for every $\gamma>0$, then conditions (3.2) and (3.3) reduce, respectively, to

$$
\begin{aligned}
& 4\left(p^{2}-\theta p\right)(p-1)-\kappa^{2}(2-p)^{2}(\sqrt{m}+m)^{2}>0, \\
& 4 p^{2}-4 \theta p-(p-2) \kappa^{2} m(m+p-2)>0 .
\end{aligned}
$$

It is easy to check that (3.5) is satisfied for every $p \geq 2$ for instance if $\theta<1 / 2$ and $\kappa m<\sqrt{8}$. On the other hand, condition (3.4) cannot be satisfied for every $p \in(1,2)$; it is satisfied, for $p \in\left[p_{0}, 2\right)$ for some $p_{0} \in(1,2)$, for instance if $4\left(p_{0}^{2}-\theta p_{0}\right)\left(p_{0}-1\right)-\kappa^{2}\left(2-p_{0}\right)^{2}(\sqrt{m}+m)^{2}>0$ and

$$
\Delta=16\left(\theta^{2}-\theta+1\right)+\kappa^{4}(m+\sqrt{m})^{4}-32 \theta \kappa^{2}(m+\sqrt{m})^{2} \leq 0 .
$$

If $\Delta>0$, then the third-order polynomial $f$, in the variable $p$, defined in (3.4) has a local maximum $f\left(p_{1}\right)$ and a local minimum $f\left(p_{2}\right)$ at some points $0<p_{1}<p_{2}$. Clearly, condition $f\left(p_{0}\right)>0$ is necessary to guarantee that $f(p)>0$ for every $p \in\left[p_{0}, 2\right)$. This condition is also sufficient if $p_{0}>p_{2}$ or $p_{1}>2$. On the other hand, when $p_{0}<p_{1}<2<p_{2}$, we also need to require that $\theta<2$ and, when $p_{0}<p_{1}<p_{2}<2$ or $p_{1}<p_{0}<p_{2} \leq 2$, we need the additional condition $f\left(p_{2}\right)>0$. Finally, if inequality (3.1) is replaced by the new condition

$$
\sum_{h, k=1}^{m}\left|\sum_{i=1}^{d} B_{h k}^{i}(x) \eta_{i}^{k}\right| \leq\left(\kappa \sqrt{v(x)}+C_{\kappa}\right) \sum_{k=1}^{m}\left\langle Q(x) \eta^{k}, \eta^{k}\right\rangle^{\frac{1}{2}}
$$

for every $x, \eta^{k} \in \mathbb{R}^{d}(k=1, \ldots, m)$ and some positive constants $\kappa$ and $C_{\kappa}$, then the generation result in Theorem 3.3 can be applied to the operator $\mathcal{A}-\lambda_{\kappa}$ for a suitable $\lambda_{\kappa}>0$. In particular, the operator $\boldsymbol{A}_{p}$ generates a strongly continuous analytic semigroup (not contractive, in general) in $L^{p}\left(\mathbb{R}^{d} ; \mathbb{R}^{m}\right)$. Indeed, condition (3.6) implies that for every $\varepsilon>0$ there exists a positive constant $\lambda$ such that Hypotheses 3.1 are satisfied with $V$ being replaced by $V+\lambda$, provided that $\varepsilon$ is chosen sufficiently small such that condition (3.2) (resp. (3.3)) holds true with $\kappa$ being replaced by $\kappa+\varepsilon$. In particular, if (3.6) is satisfied by every $\kappa>0$, then conditions (3.2) and (3.3) reduce to $\theta<p$. Hence, if $\theta<1$, then we get generation results of a family of consistent semigroups for every $p \in(1, \infty)$.

(iii) Our assumptions do not allow to cover the case when $p=1$, which will be addressed in a future paper.

In the proof of Theorem 3.3 we will take advantage of the following results.

Lemma 3.5 For every $\boldsymbol{u} \in C_{c}^{\infty}\left(\mathbb{R}^{d}, \mathbb{C}^{m}\right), \eta \in C^{1}\left(\mathbb{R}^{d}\right)$ and $\varepsilon>0$ it holds that 


$$
\begin{aligned}
\operatorname{Re} \int_{\mathbb{R}^{d}} \sum_{i=1}^{d}\left\langle B^{i} D_{i} \boldsymbol{u}, \boldsymbol{u}\right\rangle|\boldsymbol{u}|_{\varepsilon}^{p-2} \eta d x= & \frac{2-p}{2} \sum_{i=1}^{d} \int_{\mathbb{R}^{d}}\left\langle B^{i} \boldsymbol{u}, \boldsymbol{u}\right\rangle|\boldsymbol{u}|_{\varepsilon}^{p-3} \eta D_{i}|\boldsymbol{u}|_{\varepsilon} d x \\
& -\frac{1}{2} \int_{\mathbb{R}^{d}}\langle(\operatorname{div} B) \boldsymbol{u}, \boldsymbol{u}\rangle|\boldsymbol{u}|_{\varepsilon}^{p-2} \eta d x \\
& -\frac{1}{2} \int_{\mathbb{R}^{d}} \sum_{i=1}^{d}\left\langle B^{i} \boldsymbol{u}, \boldsymbol{u}\right\rangle|\boldsymbol{u}|_{\varepsilon}^{p-2} D_{i} \eta d x .
\end{aligned}
$$

Moreover,

$$
\int_{\mathbb{R}^{d}}|\boldsymbol{u}|_{\varepsilon}^{p-2} \mathfrak{q}\left(|\boldsymbol{u}|_{\varepsilon}\right) d x \leq \sum_{h=1}^{m} \int_{\mathbb{R}^{d}}|\boldsymbol{u}|_{\varepsilon}^{p-2} \mathfrak{q}\left(u_{h}\right) d x .
$$

Proof Let us first prove formula (3.7). We fix $\boldsymbol{u}, \eta$ and $\varepsilon$ as in the statement of the lemma and observe that for $p=2$, formula (3.7) can be obtained just integrating by parts and using the symmetry of the matrices $B^{i}(i=1, \ldots, d)$.

In the case $p \neq 2$, we denote by $\mathscr{I}$ the left-hand side of (3.7) and set

$$
\mathscr{K}=\frac{1}{p-2} \int_{\mathbb{R}^{d}} \sum_{i=1}^{d}\left\langle B^{i} \boldsymbol{u}, \boldsymbol{u}\right\rangle D_{i}\left(|\boldsymbol{u}|_{\varepsilon}^{p-2}\right) \eta d x=\int_{\mathbb{R}^{d}} \sum_{i=1}^{d}\left\langle B^{i} \boldsymbol{u}, \boldsymbol{u}\right\rangle|\boldsymbol{u}|_{\varepsilon}^{p-3} D_{i}|\boldsymbol{u}|_{\varepsilon} \eta d x .
$$

By integrating by parts and taking into account the symmetry of the matrices $B^{i}$, we deduce that

$$
\begin{aligned}
\mathscr{K}= & -\frac{1}{p-2} \int_{\mathbb{R}^{d}}\langle(\operatorname{div} B) \boldsymbol{u}, \boldsymbol{u}\rangle|\boldsymbol{u}|_{\varepsilon}^{p-2} \eta d x-\frac{2}{p-2} \operatorname{Re} \mathscr{I} \\
& -\frac{1}{p-2} \int_{\mathbb{R}^{d}} \sum_{i=1}^{d}\left\langle B^{i} \boldsymbol{u}, \boldsymbol{u}\right\rangle|\boldsymbol{u}|_{\varepsilon}^{p-2} D_{i} \eta d x,
\end{aligned}
$$

which immediately yields (3.7).

To prove (3.8), we preliminarily observe that, since $Q$ is symmetric, it follows that $\langle Q \zeta, \zeta\rangle=\langle Q \operatorname{Re} \zeta, \operatorname{Re} \zeta\rangle+\langle Q \operatorname{Im} \zeta, \operatorname{Im} \zeta\rangle$ whence $0 \leq\langle Q \operatorname{Re} \zeta, \operatorname{Re} \zeta\rangle \leq\langle Q \zeta, \zeta\rangle$ for every $\zeta \in \mathbb{C}^{m}$. Using this fact and Cauchy-Schwarz inequality, we obtain

$$
\begin{aligned}
\mathfrak{q}\left(|\boldsymbol{u}|_{\varepsilon}\right) & =\left(4|\boldsymbol{u}|_{\varepsilon}^{2}\right)^{-1} \mathfrak{q}\left(|\boldsymbol{u}|^{2}\right) \leq|\boldsymbol{u}|_{\varepsilon}^{-2}\left(\sum_{h=1}^{m}\left\langle Q \operatorname{Re}\left(u_{h} \nabla \bar{u}_{h}\right), \operatorname{Re}\left(u_{h} \nabla \bar{u}_{h}\right)\right\rangle^{\frac{1}{2}}\right)^{2} \\
& \leq|\boldsymbol{u}|_{\varepsilon}^{-2}\left(\sum_{h=1}^{m}\left|u_{h}\right| \mathfrak{q}\left(u_{h}\right)^{\frac{1}{2}}\right)^{2} \leq|\boldsymbol{u}|^{2}|\boldsymbol{u}|_{\varepsilon}^{-2} \sum_{h=1}^{m} \mathfrak{q}\left(u_{h}\right) .
\end{aligned}
$$

Observing that $|\boldsymbol{u}|^{2} \leq|\boldsymbol{u}|_{\varepsilon}^{2}$, estimate (3.8) follows at once from (3.9).

Recalling the definition of sectorial operator of type $S(a)$ (see, e.g., [19, Chapter I, Sect. 5.8, Definitions]), we can prove the following:

Proposition 3.6 Under the assumptions of Theorem $3.3,\left(-\mathcal{A}, C_{c}^{\infty}\left(\mathbb{R}^{d}, \mathbb{C}^{m}\right)\right)$ is sectorial of type $S(C)$ for some positive constant $C$, namely for all $\boldsymbol{u} \in C_{c}^{\infty}\left(\mathbb{R}^{d}, \mathbb{C}^{m}\right)$ 


$$
\left.\left.\left|\operatorname{Im} \int_{\mathbb{R}^{d}}\langle\mathcal{A} \boldsymbol{u}, \boldsymbol{u}\rangle\right| \boldsymbol{u}\right|^{p-2} d x\left|\leq-C \operatorname{Re} \int_{\mathbb{R}^{d}}\langle\mathcal{A} \boldsymbol{u}, \boldsymbol{u}\rangle\right| \boldsymbol{u}\right|^{p-2} d x .
$$

Proof We fix $\boldsymbol{u} \in C_{c}^{\infty}\left(\mathbb{R}^{d}, \mathbb{C}^{m}\right)$ and $\varepsilon>0$. Recalling that $D_{i}|\boldsymbol{u}|_{\varepsilon}=|\boldsymbol{u}|_{\varepsilon}^{-1} \operatorname{Re}\left\langle D_{i} \boldsymbol{u}, \boldsymbol{u}\right\rangle$, an integration by parts shows that

$$
\begin{aligned}
\operatorname{Re} & \int_{\mathbb{R}^{d}}\langle\operatorname{div}(Q \nabla \boldsymbol{u}), \boldsymbol{u}\rangle|\boldsymbol{u}|_{\varepsilon}^{p-2} d x \\
& =-\sum_{j=1}^{m} \int_{\mathbb{R}^{d}} \mathfrak{q}\left(u_{j}\right)|\boldsymbol{u}|_{\varepsilon}^{p-2} d x-(p-2) \int_{\mathbb{R}^{d}} \mathfrak{q}\left(|\boldsymbol{u}|_{\varepsilon}\right)|\boldsymbol{u}|_{\varepsilon}^{p-2} d x .
\end{aligned}
$$

Hence, using formula (3.7), splitting the term $\operatorname{Re} \sum_{i=1}^{d} \int_{\mathbb{R}^{d}}\left\langle B^{i} D_{i} \boldsymbol{u}, \boldsymbol{u}\right\rangle|\boldsymbol{u}|_{\varepsilon}^{p-2} d x$ into the sum

$$
\frac{p-2}{p} \operatorname{Re} \sum_{i=1}^{d} \int_{\mathbb{R}^{d}}\left\langle B^{i} D_{i} \boldsymbol{u}, \boldsymbol{u}\right\rangle|\boldsymbol{u}|_{\varepsilon}^{p-2} d x+\frac{2}{p} \operatorname{Re} \sum_{i=1}^{d} \int_{\mathbb{R}^{d}}\left\langle B^{i} D_{i} \boldsymbol{u}, \boldsymbol{u}\right\rangle|\boldsymbol{u}|_{\varepsilon}^{p-2} d x
$$

and, by applying (3.7) with $\eta \equiv 1$, we deduce that

$$
\begin{aligned}
- & \operatorname{Re} \int_{\mathbb{R}^{d}}\langle\mathcal{A} \boldsymbol{u}, \boldsymbol{u}\rangle|\boldsymbol{u}|_{\varepsilon}^{p-2} d x \\
= & \sum_{j=1}^{m} \int_{\mathbb{R}^{d}} \mathfrak{q}\left(u_{j}\right)|\boldsymbol{u}|_{\varepsilon}^{p-2} d x+(p-2) \int_{\mathbb{R}^{d}} \mathfrak{q}\left(|\boldsymbol{u}|_{\varepsilon}\right)|\boldsymbol{u}|_{\varepsilon}^{p-2} d x \\
& -\frac{p-2}{p} \operatorname{Re} \sum_{i=1}^{d} \int_{\mathbb{R}^{d}}\left\langle B^{i} D_{i} \boldsymbol{u}, \boldsymbol{u}\right\rangle|\boldsymbol{u}|_{\varepsilon}^{p-2} d x+\frac{p-2}{p} \sum_{i=1}^{d} \int_{\mathbb{R}^{d}}\left\langle B^{i} \boldsymbol{u}, \boldsymbol{u}\right\rangle|\boldsymbol{u}|_{\varepsilon}^{p-3} D_{i}|\boldsymbol{u}|_{\varepsilon} d x \\
& +\operatorname{Re} \int_{\mathbb{R}^{d}}\left\langle\left(p^{-1}(\operatorname{div} B)+V\right) \boldsymbol{u}, \boldsymbol{u}\right\rangle|\boldsymbol{u}|_{\varepsilon}^{p-2} d x .
\end{aligned}
$$

To ease the notation, we denote by $\mathscr{I}$ and $\mathscr{J}$ the third and fourth integral terms in the right-hand side of the previous formula. Applying (3.1), with $\eta_{i}^{k}=D_{i} u_{k}$ and $\eta_{i}^{k}=D_{i}|\boldsymbol{u}|_{\varepsilon}$ for $i=1, \ldots, d$ and $k=1, \ldots, m$, respectively, and then Cauchy-Schwarz and Hölder's inequalities, for every $\varepsilon_{0}, \varepsilon_{1}>0$ we get

$$
\begin{aligned}
\mathscr{I} & \geq-\frac{|p-2|}{p} \kappa \sum_{k=1}^{m} \int_{\mathbb{R}^{d}} \mathfrak{q}\left(u_{k}\right)^{\frac{1}{2}} v^{\frac{1}{2}}|\boldsymbol{u}||\boldsymbol{u}|_{\varepsilon}^{p-2} d x \\
& \geq-\frac{\kappa \varepsilon_{0}|p-2|}{p} \sum_{k=1}^{m} \int_{\mathbb{R}^{d}} \mathfrak{q}\left(u_{k}\right)|\boldsymbol{u}|_{\varepsilon}^{p-2} d x-\frac{\kappa m|p-2|}{4 \varepsilon_{0} p} \int_{\mathbb{R}^{d}} v|\boldsymbol{u}|^{2}|\boldsymbol{u}|_{\varepsilon}^{p-2} d x
\end{aligned}
$$

and

$$
\begin{aligned}
\mathscr{J} & \geq-\frac{|p-2|}{p} \kappa m \int_{\mathbb{R}^{d}} \mathfrak{q}\left(|\boldsymbol{u}|_{\varepsilon}\right)^{\frac{1}{2}} v^{\frac{1}{2}}|\boldsymbol{u}||\boldsymbol{u}|_{\varepsilon}^{p-2} d x \\
& \geq-\frac{\kappa m \varepsilon_{1}|p-2|}{p} \int_{\mathbb{R}^{d}} \mathfrak{q}\left(|\boldsymbol{u}|_{\varepsilon}\right)|\boldsymbol{u}|_{\varepsilon}^{p-2} d x-\frac{\kappa m|p-2|}{4 p \varepsilon_{1}} \int_{\mathbb{R}^{d}} v|\boldsymbol{u}|^{2}|\boldsymbol{u}|_{\varepsilon}^{p-2} d x .
\end{aligned}
$$

Therefore, 


$$
\begin{aligned}
-\operatorname{Re} & \int_{\mathbb{R}^{d}}\langle\mathcal{A} \boldsymbol{u}, \boldsymbol{u}\rangle|\boldsymbol{u}|_{\varepsilon}^{p-2} d x \geq\left(1-\frac{\varepsilon_{0} \kappa|p-2|}{p}\right) \sum_{j=1}^{m} \int_{\mathbb{R}^{d}} \mathfrak{q}\left(u_{j}\right)|\boldsymbol{u}|_{\varepsilon}^{p-2} d x \\
& +\left(p-2-\frac{\varepsilon_{1} \kappa m}{p}|p-2|\right) \int_{\mathbb{R}^{d}} \mathfrak{q}\left(|\boldsymbol{u}|_{\varepsilon}\right)|\boldsymbol{u}|_{\varepsilon}^{p-2} d x \\
& +\left[1-\frac{\theta}{p}-\frac{\kappa m|p-2|}{4 p}\left(\frac{1}{\varepsilon_{0}}+\frac{1}{\varepsilon_{1}}\right)\right] \int_{\mathbb{R}^{d}} v|\boldsymbol{u}|^{2}|\boldsymbol{u}|_{\varepsilon}^{p-2} d x \\
= & \left(1-\frac{\varepsilon_{0} \kappa|p-2|}{p}\right) \sum_{j=1}^{m} \int_{\mathbb{R}^{d}} \mathfrak{q}\left(u_{j}\right)|\boldsymbol{u}|_{\varepsilon}^{p-2} d x \\
& +\left(p-2-\frac{\varepsilon_{1} \kappa m}{p}|p-2|\right) \int_{\mathbb{R}^{d}} \mathfrak{q}\left(|\boldsymbol{u}|_{\varepsilon}\right)|\boldsymbol{u}|_{\varepsilon}^{p-2} d x \\
& +f_{1}\left(\varepsilon_{1}, \varepsilon_{2}\right) \int_{\mathbb{R}^{d}} v|\boldsymbol{u}|^{2}|\boldsymbol{u}|_{\varepsilon}^{p-2} d x
\end{aligned}
$$

where

$$
f_{1}\left(x_{1}, x_{2}\right)=1-\frac{\theta}{p}-\frac{\kappa m|p-2|}{4 p}\left(\frac{1}{x_{1}}+\frac{1}{x_{2}}\right), \quad x_{1}, x_{2}>0 .
$$

On the other hand, since

$$
\begin{aligned}
& \quad\left|\mathfrak{q}\left(\operatorname{Im} u_{j},|\mathbf{u}|_{\varepsilon}\right) \operatorname{Re} u_{j}\right|+\left|\mathfrak{q}\left(\operatorname{Re} u_{j},|\mathbf{u}|_{\varepsilon}\right) \operatorname{Im} u_{j}\right| \\
& \quad \leq \mathfrak{q}\left(|\mathbf{u}|_{\varepsilon}\right)^{1 / 2}\left[\mathfrak{q}\left(\operatorname{Im} u_{j}\right)^{1 / 2}\left|\operatorname{Re} u_{j}\right|+\mathfrak{q}\left(\operatorname{Re} u_{j}\right)^{1 / 2}\left|\operatorname{Im} u_{j}\right|\right] \\
& \quad \leq \mathfrak{q}\left(|\mathbf{u}|_{\varepsilon}\right)^{1 / 2}\left(\mathfrak{q}\left(\operatorname{Re} u_{j}\right)+\mathfrak{q}\left(\operatorname{Im} u_{j}\right)\right)^{1 / 2}\left(\left(\operatorname{Re} u_{j}\right)^{2}+\left(\operatorname{Im} u_{j}\right)^{2}\right)^{1 / 2} \\
& \quad=\mathfrak{q}\left(|\mathbf{u}|_{\varepsilon}\right)^{1 / 2}\left(\mathfrak{q}\left(u_{j}\right)\right)^{1 / 2}\left|u_{j}\right|
\end{aligned}
$$

for every $j=1, \ldots, m$, we can estimate

$$
\begin{aligned}
\mid \operatorname{Im} & \int_{\mathbb{R}^{d}}\langle\mathcal{A} \boldsymbol{u}, \boldsymbol{u}\rangle|\boldsymbol{u}|_{\varepsilon}^{p-2} d x \mid \\
\leq & |p-2| \sum_{j=1}^{m} \int_{\mathbb{R}^{d}}\left(\left|\mathfrak{q}\left(\operatorname{Im} u_{j},|\boldsymbol{u}|_{\varepsilon}\right) \operatorname{Re} u_{j}\right|+\left|\mathfrak{q}\left(\operatorname{Re} u_{j},|\boldsymbol{u}|_{\varepsilon}\right) \operatorname{Im} u_{j}\right|\right)|\boldsymbol{u}|_{\varepsilon}^{p-3} d x \\
& +\sum_{j, k=1}^{m} \int_{\mathbb{R}^{d}}\left|\sum_{i=1}^{d} B_{j k}^{i} D_{i} u_{k}\right||\boldsymbol{u}||\boldsymbol{u}|_{\varepsilon}^{p-2} d x+c_{1} \int_{\mathbb{R}^{d}} v|\boldsymbol{u}|^{2}|\boldsymbol{u}|_{\varepsilon}^{p-2} d x \\
\leq & \frac{|p-2|}{2} \sum_{j=1}^{m} \int_{\mathbb{R}^{d}} \mathfrak{q}\left(u_{j}\right)|\boldsymbol{u}|_{\varepsilon}^{p-2} d x+\frac{|p-2|}{2} m \int_{\mathbb{R}^{d}} \mathfrak{q}\left(|\boldsymbol{u}|_{\varepsilon}\right)|\boldsymbol{u}|_{\varepsilon}^{p-2} d x \\
& +\kappa \sum_{k=1}^{m} \int_{\mathbb{R}^{d}} \mathfrak{q}\left(u_{k}\right)^{\frac{1}{2}} v^{\frac{1}{2}}|\boldsymbol{u}||\boldsymbol{u}|_{\varepsilon}^{p-2} d x+c_{1} \int_{\mathbb{R}^{d}} v|\boldsymbol{u}|^{2}|\boldsymbol{u}|_{\varepsilon}^{p-2} d x \\
\leq & \left(\frac{|p-2|}{2}+\frac{\kappa}{2}\right) \sum_{j=1}^{m} \int_{\mathbb{R}^{d}} \mathfrak{q}\left(u_{j}\right)|\boldsymbol{u}|_{\varepsilon}^{p-2} d x+\frac{|p-2|}{2} m \int_{\mathbb{R}^{d}} \mathfrak{q}\left(|\boldsymbol{u}|_{\varepsilon}\right)|\boldsymbol{u}|_{\varepsilon}^{p-2} d x \\
& +\left(c_{1}+\frac{\kappa m}{2}\right) \int_{\mathbb{R}^{d}} v|\boldsymbol{u}|^{2}|\boldsymbol{u}|_{\varepsilon}^{p-2} d x .
\end{aligned}
$$


We now distinguish between the cases $p \in(1,2)$ and $p \geq 2$. In the first case, the coefficient of the second term in the last side of (3.13) is negative. Using inequality (3.8), we can continue estimate (3.13) and get

$$
\begin{array}{r}
-\operatorname{Re} \int_{\mathbb{R}^{d}}\langle\mathcal{A} \boldsymbol{u}, \boldsymbol{u}\rangle|\boldsymbol{u}|_{\varepsilon}^{p-2} d x \geq \\
g_{1}\left(\varepsilon_{0}, \varepsilon_{1}\right) \sum_{j=1}^{m} \int_{\mathbb{R}^{d}} \mathfrak{q}\left(u_{j}\right)|\boldsymbol{u}|_{\varepsilon}^{p-2} d x \\
+f_{1}\left(\varepsilon_{0}, \varepsilon_{1}\right) \int_{\mathbb{R}^{d}} v|\boldsymbol{u}|^{2}|\boldsymbol{u}|_{\varepsilon}^{p-2} d x
\end{array}
$$

where

$$
g_{1}\left(x_{1}, x_{2}\right)=p-1+\frac{p-2}{p} \kappa\left(x_{1}+m x_{2}\right)
$$

for every $x_{1}, x_{2}>0$, and, similarly,

$$
\begin{aligned}
& \left.\left|\operatorname{Im} \int_{\mathbb{R}^{d}}\langle\mathcal{A} \boldsymbol{u}, \boldsymbol{u}\rangle\right| \boldsymbol{u}\right|_{\varepsilon} ^{p-2} d x \mid \\
& \quad \leq\left(\frac{2-p}{2}+\frac{\kappa}{2}+\frac{2-p}{2} m\right) \sum_{j=1}^{m} \int_{\mathbb{R}^{d}} \mathfrak{q}\left(u_{j}\right)|\boldsymbol{u}|_{\varepsilon}^{p-2} d x+\left(c_{1}+\frac{\kappa m}{2}\right) \int_{\mathbb{R}^{d}} v|\boldsymbol{u}|^{2}|\boldsymbol{u}|_{\varepsilon}^{p-2} d x .
\end{aligned}
$$

The supremum of function $f_{1}$, subject to the constraint $g_{1}\left(x_{1}, x_{2}\right)>0$, is

$$
1-\frac{\theta}{p}-\frac{\kappa^{2}(p-2)^{2} m(1+\sqrt{m})^{2}}{4 p^{2}(p-1)},
$$

see Subsection A.3, which is positive thanks to condition (3.2). Then, we can choose $\varepsilon_{0}$ and $\varepsilon_{1}$ positive and such that the coefficients of the two terms in the right-hand side of (3.15) are both positive. Thus, we get

$$
\left.\left.\left|\operatorname{Im} \int_{\mathbb{R}^{d}}\langle\mathcal{A} \boldsymbol{u}, \boldsymbol{u}\rangle\right| \boldsymbol{u}\right|_{\varepsilon} ^{p-2} d x\left|\leq-C \operatorname{Re} \int_{\mathbb{R}^{d}}\langle\mathcal{A} \boldsymbol{u}, \boldsymbol{u}\rangle\right| \boldsymbol{u}\right|_{\varepsilon} ^{p-2} d x
$$

with

$$
C \geq \max \left\{\left(\frac{2-p}{2}+\frac{\kappa}{2}+\frac{2-p}{2} m\right) \frac{1}{g_{1}\left(\varepsilon_{0}, \varepsilon_{1}\right)},\left(c_{1}+\frac{\kappa m}{2}\right) \frac{1}{f_{1}\left(\varepsilon_{0}, \varepsilon_{1}\right)}\right\} .
$$

Now, we address the case $p \geq 2$. Here, the coefficient of the second term in the right-hand side of (3.13) can be made positive by choosing $\varepsilon_{1}$ small enough. Note that the supremum of the function $f_{1}$, subject to the constraints $x_{1} \in(0, p /(\kappa(p-2)))$ and $x_{2} \in(0, p /(\kappa m))$, is

$$
1-\frac{\theta}{p}-\frac{p-2}{4 p^{2}} \kappa^{2} m(m+p-2)
$$

(see Subsect. A.4), which is positive due to condition (3.3). Hence, we can determine $\varepsilon_{0}$ and $\varepsilon_{1}$ such that the coefficients of the three terms in the right-hand side of (3.13) are all positive. With this choice of the parameters, estimate (3.17) follows immediately with 


$$
C \geq \max \left\{\frac{p(p-2+\kappa)}{2\left(p-\varepsilon_{0} \kappa(p-2)\right)}, \frac{m p}{2\left(p-\varepsilon_{1} \kappa m\right)},\left(c_{1}+\frac{\kappa m}{2}\right) \frac{1}{f_{1}\left(\varepsilon_{0}, \varepsilon_{1}\right)}\right\} .
$$

Finally, letting $\varepsilon$ tend to $0^{+}$in (3.17), by dominated convergence we get (3.10) in both cases.

Proposition 3.7 Under the assumptions of Theorem 3.3, assume that $C_{\gamma}=0$ in Hypothesis $3.1(v)$. Then, there exists a constant $C=C(m, p, \gamma, \kappa, \theta)>0$ such that

$$
\|v \boldsymbol{u}\|_{p} \leq C\|\mathcal{A} \boldsymbol{u}\|_{p}, \quad \boldsymbol{u} \in C_{c}^{\infty}\left(\mathbb{R}^{d} ; \mathbb{C}^{m}\right) .
$$

Proof Since the coefficients of the operator $\mathcal{A}$ are real-valued, we can limit ourselves to considering functions with values in $\mathbb{R}^{m}$. We fix $\boldsymbol{u} \in C_{c}^{\infty}\left(\mathbb{R}^{d} ; \mathbb{R}^{m}\right), \varepsilon>0$ and set $\boldsymbol{f}=-\mathcal{A} \boldsymbol{u}$. Then,

$$
\begin{aligned}
\int_{\mathbb{R}^{d}}\langle\boldsymbol{f}, \boldsymbol{u}\rangle|\boldsymbol{u}|_{\varepsilon}^{p-2} v^{p-1} d x= & -\int_{\mathbb{R}^{d}}\langle\operatorname{div}(Q \nabla \boldsymbol{u}), \boldsymbol{u}\rangle|\boldsymbol{u}|_{\varepsilon}^{p-2} v^{p-1} d x \\
& -\int_{\mathbb{R}^{d}} \sum_{i=1}^{d}\left\langle B^{i} D_{i} \boldsymbol{u}, \boldsymbol{u}\right\rangle|\boldsymbol{u}|_{\varepsilon}^{p-2} v^{p-1} d x \\
& +\int_{\mathbb{R}^{d}}\langle V \boldsymbol{u}, \boldsymbol{u}\rangle|\boldsymbol{u}|_{\varepsilon}^{p-2} v^{p-1} d x=\mathscr{J}_{1}+\mathscr{J}_{2}+\mathscr{J}_{3} .
\end{aligned}
$$

Integrating by parts, taking (3.11) into account, we deduce that

$$
\begin{aligned}
\mathscr{J}_{1}= & \sum_{j=1}^{m} \int_{\mathbb{R}^{d}} \mathfrak{q}\left(u_{j}\right)|\boldsymbol{u}|_{\varepsilon}^{p-2} v^{p-1} d x+(p-2) \int_{\mathbb{R}^{d}} \mathfrak{q}\left(|\boldsymbol{u}|_{\varepsilon}\right) v^{p-1}|\boldsymbol{u}|_{\varepsilon}^{p-2} d x \\
& +\sum_{j=1}^{m} \int_{\mathbb{R}^{d}} \mathfrak{q}\left(u_{j}, v\right) u_{j} v^{p-2}|\boldsymbol{u}|_{\varepsilon}^{p-2} d x+(p-2) \int_{\mathbb{R}^{d}} \mathfrak{q}\left(|\boldsymbol{u}|_{\varepsilon}, v\right) v^{p-2}|\boldsymbol{u}|_{\varepsilon}^{p-1} d x
\end{aligned}
$$

Thus, applying Cauchy-Schwarz and Hölder's inequalities and taking Hypothesis 3.1(v) into account, we get

$$
\begin{aligned}
\mathscr{J}_{1} \geq & \left(1-\varepsilon_{1}\right) \sum_{j=1}^{m} \int_{\mathbb{R}^{d}} \mathfrak{q}\left(u_{j}\right)|\boldsymbol{u}|_{\varepsilon}^{p-2} v^{p-1} d x-\left(\frac{1}{4 \varepsilon_{1}}+\frac{|p-2|}{4 \varepsilon_{2}}\right) \gamma^{2} \int_{\mathbb{R}^{d}} v^{p}|\boldsymbol{u}|_{\varepsilon}^{p} d x \\
& +\left(p-2-\varepsilon_{2}|p-2|\right) \int_{\mathbb{R}^{d}} \mathfrak{q}\left(|\boldsymbol{u}|_{\varepsilon}\right) v^{p-1}|\boldsymbol{u}|_{\varepsilon}^{p-2} d x
\end{aligned}
$$

for every $\varepsilon_{1}, \varepsilon_{2}>0$.

We now estimate the term $\mathscr{J}_{2}$. Using the same arguments as in the proof of (3.12) and applying Young's inequality, we get 


$$
\begin{aligned}
\mathscr{J}_{2} \geq & -\frac{|p-2|}{p} \kappa \sum_{k=1}^{m} \int_{\mathbb{R}^{d}} \mathfrak{q}\left(u_{k}\right)^{\frac{1}{2}}|\boldsymbol{u}||\boldsymbol{u}|_{\varepsilon}^{p-2} v^{p-\frac{1}{2}} d x \\
& -\frac{|p-2|}{p} \kappa m \int_{\mathbb{R}^{d}} \mathfrak{q}\left(|\boldsymbol{u}|_{\varepsilon}\right)^{\frac{1}{2}}|\boldsymbol{u}||\boldsymbol{u}|_{\varepsilon}^{p-2} v^{p-\frac{1}{2}} d x+\frac{1}{p} \int_{\mathbb{R}^{d}}\langle(\operatorname{div} B) \boldsymbol{u}, \boldsymbol{u}\rangle|\boldsymbol{u}|_{\varepsilon}^{p-2} v^{p-1} d x \\
& -\frac{p-1}{p} \kappa m \int_{\mathbb{R}^{d}} \mathfrak{q}(v)^{\frac{1}{2}} v^{p-\frac{3}{2}}|\boldsymbol{u}|^{2}|\boldsymbol{u}|_{\varepsilon}^{p-2} d x \\
\geq & -\kappa \frac{|p-2|}{p} \varepsilon_{3} \sum_{k=1}^{m} \int_{\mathbb{R}^{d}} \mathfrak{q}\left(u_{k}\right)|\boldsymbol{u}|_{\varepsilon}^{p-2} v^{p-1} d x \\
& -\frac{|p-2|}{p} \kappa m \varepsilon_{4} \int_{\mathbb{R}^{d}} \mathfrak{q}\left(|\boldsymbol{u}|_{\varepsilon}\right)|\boldsymbol{u}|_{\varepsilon}^{p-2} v^{p-1} d x+\frac{1}{p} \int_{\mathbb{R}^{d}}\langle(\operatorname{div} B) \boldsymbol{u}, \boldsymbol{u}\rangle|\boldsymbol{u}|_{\varepsilon}^{p-2} v^{p-1} d x \\
& -\left[\frac{\kappa|p-2|}{4 p} m\left(\frac{1}{\varepsilon_{3}}+\frac{1}{\varepsilon_{4}}\right)+\frac{\gamma \kappa m(p-1)}{p}\right] \int_{\mathbb{R}^{d}}|\boldsymbol{u}|^{2}|\boldsymbol{u}|_{\varepsilon}^{p-2} v^{p} d x
\end{aligned}
$$

for every $\varepsilon_{3}, \varepsilon_{4}>0$.

Summing up, we have proved that

$$
\begin{aligned}
& \int_{\mathbb{R}^{d}}\langle\boldsymbol{f}, \boldsymbol{u}\rangle|\boldsymbol{u}|_{\varepsilon}^{p-2} v^{p-1} d x \geq\left(1-\varepsilon_{1}-\kappa \frac{|p-2|}{p} \varepsilon_{3}\right) \sum_{k=1}^{m} \int_{\mathbb{R}^{d}} \mathfrak{q}\left(u_{k}\right)|\boldsymbol{u}|_{\varepsilon}^{p-2} v^{p-1} d x \\
& \quad+\left[p-2-|p-2|\left(\varepsilon_{2}+\frac{\kappa m}{p} \varepsilon_{4}\right)\right] \int_{\mathbb{R}^{d}} \mathfrak{q}\left(|\boldsymbol{u}|_{\varepsilon}\right)|\boldsymbol{u}|_{\varepsilon}^{p-2} v^{p-1} d x \\
& \quad+f_{2}\left(\varepsilon_{1}, \varepsilon_{2}, \varepsilon_{3}, \varepsilon_{4}\right) \int_{\mathbb{R}^{d}} v^{p}|\boldsymbol{u}|^{2}|\boldsymbol{u}|_{\varepsilon}^{p-2} d x
\end{aligned}
$$

where

$$
f_{2}\left(x_{1}, x_{2}, x_{3}, x_{4}\right)=1-\frac{\theta}{p}-\gamma^{2}\left(\frac{1}{4 x_{1}}+\frac{|p-2|}{4 x_{2}}\right)-\frac{\kappa m|p-2|}{4 p}\left(\frac{1}{x_{3}}+\frac{1}{x_{4}}\right)-\frac{p-1}{p} \kappa m \gamma
$$

for every $\left(x_{1}, x_{2}, x_{3}, x_{4}\right) \in \mathbb{R}_{+}^{4}$. Combining (3.19) with the estimate

$$
\int_{\mathbb{R}^{d}}\langle\boldsymbol{f}, \boldsymbol{u}\rangle|\boldsymbol{u}|_{\varepsilon}^{p-2} v^{p-1} d x \leq \delta \int_{B(0, R)} v^{p}|\boldsymbol{u}|_{\varepsilon}^{p} d x+C \int_{\mathbb{R}^{d}}|\boldsymbol{f}|^{p} d x,
$$

which holds true for every $\delta>0$ and some positive constant $C=C(\delta, p)$, where $B(0, R)$ is any ball containing the support of the function $f$, we get

$$
\begin{aligned}
& \left(1-\varepsilon_{1}-\kappa \frac{|p-2|}{p} \varepsilon_{3}\right) \sum_{k=1}^{m} \int_{\mathbb{R}^{d}} \mathfrak{q}\left(u_{k}\right)|\boldsymbol{u}|_{\varepsilon}^{p-2} v^{p-1} d x \\
& +\left[p-2-|p-2|\left(\varepsilon_{2}+\frac{\kappa m}{p} \varepsilon_{4}\right)\right] \int_{\mathbb{R}^{d}} \mathfrak{q}\left(|\boldsymbol{u}|_{\varepsilon}\right)|\boldsymbol{u}|_{\varepsilon}^{p-2} v^{p-1} d x \\
& \quad+f_{2}\left(\varepsilon_{1}, \varepsilon_{2}, \varepsilon_{3}, \varepsilon_{4}\right) \int_{\mathbb{R}^{d}}|\boldsymbol{u}|^{2}|\boldsymbol{u}|_{\varepsilon}^{p-2} v^{p} d x-\delta\left\|v|\boldsymbol{u}|_{\varepsilon}\right\|_{L^{p}(B(0, R))}^{p} \leq C \int_{\mathbb{R}^{d}}|\boldsymbol{f}|^{p} d x
\end{aligned}
$$

As in Proposition 3.6, we distinguish between the cases $p \in(1,2)$ and $p \geq 2$. In the first case, assuming that $1-\varepsilon_{1}-p^{-1} \kappa|p-2| \varepsilon_{3}>0$ and using (3.9), we can combine the first two terms in the left-hand side of (3.21) and obtain the inequality 


$$
\begin{aligned}
& g_{2}\left(\varepsilon_{1}, \varepsilon_{2}, \varepsilon_{3}, \varepsilon_{4}\right) \int_{\mathbb{R}^{d}} \mathfrak{q}\left(|\boldsymbol{u}|_{\varepsilon}\right)|\boldsymbol{u}|_{\varepsilon}^{p-2} v^{p-1} d x \\
& \quad+f_{2}\left(\varepsilon_{1}, \varepsilon_{2}, \varepsilon_{3}, \varepsilon_{4}\right) \int_{\mathbb{R}^{d}}|\boldsymbol{u}|^{2}|\boldsymbol{u}|_{\varepsilon}^{p-2} v^{p} d x-\delta\left\|v|\boldsymbol{u}|_{\varepsilon}\right\|_{L^{p}(B(0, R))}^{p} \leq C \int_{\mathbb{R}^{d}}|\boldsymbol{f}|^{p} d x,
\end{aligned}
$$

where the function $g_{2}: \mathbb{R}_{+}^{4} \rightarrow \mathbb{R}$ is defined by

$$
g_{2}\left(x_{1}, x_{2}, x_{3}, x_{4}\right)=p-1-x_{1}-\kappa \frac{|p-2|}{p} x_{3}-(2-p)\left(x_{2}+\frac{\kappa m}{p} x_{4}\right)
$$

for every $\left(x_{1}, x_{2}, x_{3}, x_{4}\right) \in \mathbb{R}_{+}^{4}$. It is easy to check that the supremum of $f_{2}$, subject to the constraint $g_{2}\left(x_{1}, \ldots, x_{4}\right)>0$, is given by

$$
1-\frac{\theta}{p}-\frac{p-1}{p} \kappa m \gamma-\frac{1}{4(p-1)}\left[(3-p) \gamma+\frac{\kappa|p-2|}{p}(\sqrt{m}+m)\right]^{2},
$$

(see Subsect. A.1 for further details). Due to condition (3.2), this supremum is positive. Thus, we can choose the parameters $\varepsilon_{j}(j=1, \ldots, 4)$ such that the coefficients of the two first integral terms in the left-hand side of (3.22) are both positive, so that

$$
f_{2}\left(\varepsilon_{1}, \varepsilon_{2}, \varepsilon_{3}, \varepsilon_{4}\right) \int_{\mathbb{R}^{d}}|\boldsymbol{u}|^{2}|\boldsymbol{u}|_{\varepsilon}^{p-2} v^{p} d x-\delta\left\|v|\boldsymbol{u}|_{\varepsilon}\right\|_{L^{p}(B(0, R))}^{p} \leq C \int_{\mathbb{R}^{d}}|\boldsymbol{f}|^{p} d x .
$$

Letting $\varepsilon$ tend to $0^{+}$, we can choose $\delta>0$ such that estimate (3.18) follows.

If $p \geq 2$, then the supremum of the function $f_{2}$, subject to the constraints $p\left(1-x_{1}\right)-\kappa(p-2) x_{3}>0$ and $p\left(1-x_{2}\right)-\kappa m x_{4}>0$, is

$$
1-\frac{\theta}{p}-\frac{p-1}{p} \kappa m \gamma-\frac{\gamma^{2}}{4}(p-1)-\frac{p-2}{2 p} \kappa \gamma(\sqrt{m}+m)-\frac{p-2}{4 p^{2}} \kappa^{2} m(m+p-2),
$$

(see Subsect. A.2 for further details), which is positive due to condition (3.3). Now, we can argue as in the case $p \in(1,2)$ to obtain estimate (3.18).

Proof (Proof of theorem 3.3) The proof is articulated in some steps.

Step 1. Here, we prove that there exist positive constants $M_{1}$ and $M_{2}$ depending on $\kappa, c_{0}, c_{1}, c_{2}, \gamma, C_{\gamma}, \theta$ and $p$, such that

$$
M_{1}\|\boldsymbol{u}\|_{D_{p}} \leq\|\mathcal{A} \boldsymbol{u}-\boldsymbol{u}\|_{p} \leq M_{2}\|\boldsymbol{u}\|_{D_{p}}, \quad \boldsymbol{u} \in C_{c}^{\infty}\left(\mathbb{R}^{d} ; \mathbb{C}^{m}\right) .
$$

Also in this case, we can assume that $\boldsymbol{u}$ takes values in $\mathbb{R}^{m}$. Adapting the arguments in [29, Lemma 2.5, Proposition 3.3] and using Hypothesis 3.1(v), we can show that, for every $\varepsilon>0$, there exists a positive constant $K_{\varepsilon}$, depending also on $p$ and the constants $\kappa, c_{0}, c_{1}, c_{2}, \gamma, C_{\gamma}$, such that ${ }^{1}$

$$
\left\|\sum_{i=1}^{d} B^{i} D_{i} \boldsymbol{u}\right\|_{p} \leq \varepsilon\left\|\mathcal{A}_{0} \boldsymbol{u}\right\|_{p}+K_{\varepsilon}\|V \boldsymbol{u}\|_{p}, \quad \boldsymbol{u} \in C_{c}^{\infty}\left(\mathbb{R}^{d} ; \mathbb{R}^{m}\right) .
$$

\footnotetext{
${ }^{1}$ When $p \in(1,2)$ the additional condition $\gamma^{2}<4(p-1)^{-1}$ is needed to apply the arguments in [29]. Note that this condition is a straightforward consequence of (3.2), since this latter condition implies that $1-(3-p)^{2} \gamma^{2} /(4(p-1))>0$.
} 
We first assume that $C_{\gamma}=0$. Using inequality (3.25) and taking into account Proposition 3.7, we can estimate

$$
\begin{aligned}
\|\boldsymbol{u}\|_{D_{p}} & \leq\|\mathcal{A} \boldsymbol{u}\|_{p}+\left\|\sum_{i=1}^{d} B^{i} D_{i} \boldsymbol{u}\right\|_{p}+\|V \boldsymbol{u}\|_{p}+\|v \boldsymbol{u}\|_{p} \\
& \leq\|\mathcal{A} \boldsymbol{u}\|_{p}+\frac{1}{2}\left\|\mathcal{A}_{0} \boldsymbol{u}\right\|_{p}+\left[\left(1+K_{1 / 2}\right) c_{1}+1\right]\|v \boldsymbol{u}\|_{p} \\
& \leq \frac{1}{2}\|\boldsymbol{u}\|_{D_{p}}+\left[1+\left(1+K_{1 / 2}\right) c_{1} C+\frac{C}{2}\right]\|\mathcal{A} \boldsymbol{u}\|_{p}
\end{aligned}
$$

for every $\boldsymbol{u} \in C_{c}^{\infty}\left(\mathbb{R}^{d} ; \mathbb{R}^{m}\right)$, so that using Proposition 3.6, which shows that the operator $\mathcal{A}$ is dissipative, we get $\|\boldsymbol{u}\|_{D_{p}} \leq 2 K\|\mathcal{A} \boldsymbol{u}\|_{p} \leq 2 K\|\mathcal{A} \boldsymbol{u}-\boldsymbol{u}\|_{p}+2 K\|\boldsymbol{u}\|_{p}, \leq 4 K\|\mathcal{A} \boldsymbol{u}-\boldsymbol{u}\|_{p}$, where $K=1+\left(1+K_{1 / 2}\right) c_{1} C+C / 2$. The first part of (3.24) follows with $M_{1}=(4 K)^{-1}$. If $C_{\gamma} \neq 0$, then we can determine a positive constant $\lambda$ such that $V+\lambda I$ and $v+\lambda$ satisfy Hypothesis 3.1(v) with $C_{\gamma}=0$. In such a case, using again the dissipativity of $\mathcal{A}$ we obtain

$$
\|\boldsymbol{u}\|_{D_{p}} \leq \tilde{K}\|\mathcal{A} \boldsymbol{u}-\lambda \boldsymbol{u}-\boldsymbol{u}\|_{p} \leq \widetilde{K}\|\mathcal{A} \boldsymbol{u}-\boldsymbol{u}\|_{p}+\widetilde{K} \lambda\|\boldsymbol{u}\|_{p} \leq(1+\lambda) \widetilde{K}\|\mathcal{A} \boldsymbol{u}-\boldsymbol{u}\|_{p}
$$

and the first part of (3.24) follows with $M_{1}=((1+\lambda) \widetilde{K})^{-1}$ and $\widetilde{K}$ is a positive constant, depending on $c_{1}, K_{1 / 2}$ and $C$.

To prove the other part of (3.24) we argue similarly, observing that

$$
\begin{aligned}
\|\mathcal{A} \boldsymbol{u}-\boldsymbol{u}\|_{p} & \leq 2\left\|\mathcal{A}_{0} \boldsymbol{u}\right\|_{p}+\left(K_{1}+1\right)\|V \boldsymbol{u}\|_{p}+\|\boldsymbol{u}\|_{p} \\
& \leq 2\left\|\mathcal{A}_{0} \boldsymbol{u}\right\|_{p}+\left(c_{1} K_{1}+c_{1}+c_{0}^{-1}\right)\|v \boldsymbol{u}\|_{p} \\
& \leq \max \left\{2, c_{1} K_{1}+c_{1}+c_{0}^{-1}\right\}\|\boldsymbol{u}\|_{D_{p}} .
\end{aligned}
$$

Step 2. Here, we prove that $D_{p}=D_{p, \max }\left(\mathcal{A}_{0}-V\right):=\left\{\boldsymbol{u} \in L^{p}\left(\mathbb{R}^{d} ; \mathbb{C}^{m}\right): \mathcal{A}_{0} \boldsymbol{u}-V \boldsymbol{u} \in L^{p}\left(\mathbb{R}^{d} ; \mathbb{C}^{m}\right)\right\}$ and that $C_{c}^{\infty}\left(\mathbb{R}^{d} ; \mathbb{R}^{m}\right)$ is dense in $D_{p}$. Clearly, $D_{p} \subset D_{p \text {,max }}\left(\mathcal{A}_{0}-V\right)$, so let us prove the other inclusion. We fix $\boldsymbol{u} \in D_{p \text {,max }}\left(\mathcal{A}_{0}-V\right)$ and observe that Theorem 2.3 guarantees the existence of a sequence $\left(\boldsymbol{u}_{n}\right) \subset C_{c}^{\infty}\left(\mathbb{R}^{d} ; \mathbb{R}^{m}\right)$ converging to $\boldsymbol{u}$ in $L^{p}\left(\mathbb{R}^{d} ; \mathbb{R}^{m}\right)$ and such that $\mathcal{A}_{0} \boldsymbol{u}_{n}-V \boldsymbol{u}_{n}$ converges to $\mathcal{A}_{0} \boldsymbol{u}-V \boldsymbol{u}$ in $L^{p}\left(\mathbb{R}^{d} ; \mathbb{R}^{m}\right)$ as $n$ tends to $\infty$. Step 1 , applied with $B^{i}=0$, shows that $\left(\boldsymbol{u}_{n}\right)$ is a Cauchy sequence in $D_{p}$ endowed with the norm $\|\cdot\|_{D_{p}}$. Since this latter is a Banach space, we conclude that $\boldsymbol{u} \in D_{p}$ and the inclusion $D_{p, \max }\left(\mathcal{A}_{0}-V\right) \subset D_{p}$ follows. This argument also shows that $C_{c}^{\infty}\left(\mathbb{R}^{d} ; \mathbb{R}^{m}\right)$ is dense in $D_{p}$.

Step 3. Here, we prove that the operator $\left(\mathcal{A}, D_{p}\right)$ generates an analytic contraction semigroup in $L^{p}\left(\mathbb{R}^{d} ; \mathbb{C}^{m}\right)$. In view of Proposition 3.6, [19, Theorem 1.5.9], it suffices to show that the operator $I-\mathcal{A}: D_{p} \rightarrow L^{p}\left(\mathbb{R}^{d} ; \mathbb{C}^{m}\right)$ is surjective. For this purpose, we apply the continuity method (see, e.g., [18, Theorem 5.2]). For every $t \in[0,1]$, we introduce the operator

$$
\mathcal{L}_{t} \boldsymbol{u}=\boldsymbol{u}-\operatorname{div}(Q \nabla \boldsymbol{u})-t \sum_{i=1}^{d} B^{i} D_{i} \boldsymbol{u}+V \boldsymbol{u}, \quad \boldsymbol{u} \in D_{p} .
$$

Due to the density of $C_{c}^{\infty}\left(\mathbb{R}^{d} ; \mathbb{R}^{m}\right)$ in $D_{p}$, we can first extend (3.25) to every $\boldsymbol{u} \in D_{p}$ and, then, using this inequality, we can extend (3.24) to every $\boldsymbol{u} \in D_{p}$. Thus, we can determine a positive constant $K$, independent of $t \in[0,1]$, such that $\left\|\mathcal{L}_{t} \boldsymbol{u}\right\|_{p} \geq K\|\boldsymbol{u}\|_{D_{p}}$ for every $t \in[0,1]$ and $\boldsymbol{u} \in D_{p}$. Moreover, Theorem 2.3 and Step 2 show that the operator $\left(\mathcal{A}_{0}-V, D_{p}\right)$ generates a strongly continuous semigroup of contractions in $L^{p}\left(\mathbb{R}^{d} ; \mathbb{C}^{m}\right)$. 
Hence, the operator $\mathcal{L}_{0}$ is surjective on $L^{p}\left(\mathbb{R}^{d} ; \mathbb{C}^{m}\right)$. Since the operator $\mathcal{A}_{0}$ has real-valued coefficients, it follows that $\mathcal{L}_{0}$ is surjective on $L^{p}\left(\mathbb{R}^{d} ; \mathbb{C}^{m}\right)$. The continuity method applies showing that also the operator $\mathcal{L}_{1}=I-\mathcal{A}: D_{p} \rightarrow L^{p}\left(\mathbb{R}^{d} ; \mathbb{C}^{m}\right)$ is surjective.

Step 4. Finally we complete the proof, showing that, if $q$ is a different index in $(1, \infty)$, which satisfies the assumptions of the theorem, then $\boldsymbol{T}_{p}(t) \boldsymbol{f}=\boldsymbol{T}_{q}(t) \boldsymbol{f}$ for every $t>0$ and $\boldsymbol{f} \in L^{p}\left(\mathbb{R}^{d} ; \mathbb{C}^{m}\right) \cap L^{q}\left(\mathbb{R}^{d} ; \mathbb{C}^{m}\right)$. Since both $\boldsymbol{T}_{p}(t)$ and $\boldsymbol{T}_{q}(t)$ map functions with values in $\mathbb{R}^{m}$ in functions with values in $\mathbb{R}^{m}$, we can limit ourselves to considering functions with values in $\mathbb{R}^{m}$. By Theorem 2.3, the semigroups generated by the closure of the operator $\left(\mathcal{A}_{0}-V, C_{c}^{\infty}\left(\mathbb{R}^{d} ; \mathbb{R}^{m}\right)\right)$ in $L^{p}\left(\mathbb{R}^{d} ; \mathbb{R}^{m}\right)$ and in $L^{q}\left(\mathbb{R}^{d} ; \mathbb{R}^{m}\right)$, coincide on $L^{p}\left(\mathbb{R}^{d} ; \mathbb{R}^{m}\right) \cap L^{q}\left(\mathbb{R}^{d} ; \mathbb{R}^{m}\right)$. As a by-product, writing the resolvent operators as the Laplace transform of the semigroups, we infer that the resolvent operators coincide on $L^{p}\left(\mathbb{R}^{d} ; \mathbb{R}^{m}\right) \cap L^{q}\left(\mathbb{R}^{d} ; \mathbb{R}^{m}\right)$. Therefore, for every $f \in L^{p}\left(\mathbb{R}^{d} ; \mathbb{R}^{m}\right) \cap L^{q}\left(\mathbb{R}^{d} ; \mathbb{R}^{m}\right)$ and $\lambda \in \mathbb{R}$ sufficiently large there exists a unique $\boldsymbol{u} \in D_{p} \cap D_{q}$ which solves the equation $\lambda \boldsymbol{u}-\mathcal{A}_{0} \boldsymbol{u}+V \boldsymbol{u}=\boldsymbol{f}$.

Next, we observe that all the computations in the previous steps can be performed replacing $\|\cdot\|_{D_{p}}$ with $\|\cdot\|_{D_{p}}+\|\cdot\|_{D_{q}}$ and by applying the method of continuity in the space $L^{p}\left(\mathbb{R}^{d} ; \mathbb{R}^{m}\right) \cap L^{q}\left(\mathbb{R}^{d} ; \mathbb{R}^{m}\right)$ endowed with the norm $\|\cdot\|_{p}+\|\cdot\|_{q}$. It follows that $\lambda I-\mathcal{A}: D_{p} \cap D_{q} \rightarrow L^{p}\left(\mathbb{R}^{d} ; \mathbb{R}^{m}\right) \cap L^{q}\left(\mathbb{R}^{d} ; \mathbb{R}^{m}\right)$ is invertible for every $\lambda>0$, and therefore $\left(\lambda I-\boldsymbol{A}_{p}\right)^{-1} \boldsymbol{f}=\left(\lambda I-\boldsymbol{A}_{q}\right)^{-1} \boldsymbol{f}$ for every $\boldsymbol{f} \in L^{p}\left(\mathbb{R}^{d} ; \mathbb{R}^{m}\right) \cap L^{q}\left(\mathbb{R}^{d} ; \mathbb{R}^{m}\right)$. By the representation formula of semigroups in terms of the resolvents, we get the assertion.

\section{Remark 3.8}

(i) It is worth observing explicitly that estimate (3.25) does not imply that the drift term is a small perturbation of $\mathcal{A}_{0}$ or $\mathcal{A}_{0}-V$, see [31, Remark 3.6].

(ii) We point out that if there exists $\mu_{0}>0$ such that $\langle Q(x) \xi, \xi\rangle \geq \mu_{0}|\xi|^{2}$ for every $x, \xi \in \mathbb{R}^{d}$, then $D_{2}$ is continuously embedded in $W^{1,2}\left(\mathbb{R}^{d} ; \mathbb{R}^{m}\right)$. Indeed, from (3.21), with $v \equiv 1$, it follows that

$$
\sum_{k=1}^{m} \int_{\mathbb{R}^{d}} \mathfrak{q}\left(u_{k}\right) d x \leq C^{\prime} \int_{\mathbb{R}^{d}}|\mathcal{A} \boldsymbol{u}|^{2} d x, \quad \boldsymbol{u} \in C_{c}^{\infty}\left(\mathbb{R}^{d} ; \mathbb{C}^{m}\right),
$$

from which it follows immediately that

$$
\int_{\mathbb{R}^{d}}|\nabla \boldsymbol{u}|^{2} d x \leq C^{\prime} \mu_{0}^{-1} \int_{\mathbb{R}^{d}}|\mathcal{A} \boldsymbol{u}|^{2} d x .
$$

Since $C_{c}^{\infty}\left(\mathbb{R}^{d} ; \mathbb{C}^{m}\right)$ is a core for $\boldsymbol{A}_{2}$, the previous inequality extends to every $\boldsymbol{u} \in D_{2}$.

To conclude this section we prove that $D_{p}$ coincides with the maximal domain of the realization $\boldsymbol{A}_{p}$ of $\mathcal{A}$ in $L^{p}\left(\mathbb{R}^{d} ; \mathbb{R}^{m}\right)$.

Proposition 3.9 Fix $p \in(1, \infty)$ such that the assumptions of Theorem 3.3 are satisfied. Then,

$$
D_{p}=\left\{\boldsymbol{u} \in L^{p}\left(\mathbb{R}^{d} ; \mathbb{C}^{m}\right) \cap W_{\mathrm{loc}}^{2, p}\left(\mathbb{R}^{d} ; \mathbb{C}^{m}\right): \mathcal{A} \boldsymbol{u} \in L^{p}\left(\mathbb{R}^{d} ; \mathbb{C}^{m}\right)\right\}=: D_{\max }\left(\boldsymbol{A}_{p}\right)
$$

Consequently, $C_{c}^{\infty}\left(\mathbb{R}^{d} ; \mathbb{C}^{m}\right)$ is a core for $\left(\boldsymbol{A}_{p}, D_{\max }\left(\boldsymbol{A}_{p}\right)\right)$. 
Proof First of all, let us observe that the inclusion $D_{p} \subset D_{\max }\left(\mathcal{A}_{p}\right)$ is immediate consequence of the estimate $\|\boldsymbol{u}\|_{p} \leq c_{0}^{-1}\|v \boldsymbol{u}\|_{p}$ and interpolative estimate (3.25). To prove that $D_{\max }\left(\mathcal{A}_{p}\right) \subset D_{p}$, it suffices to prove that $\lambda I-\mathcal{A}$ is injective on $D_{\text {max }}\left(\mathcal{A}_{p}\right)$ for some (hence all) $\lambda>0$. So, let us consider $\boldsymbol{u} \in D_{\max }\left(\mathcal{A}_{p}\right)$ such that $\lambda \boldsymbol{u}-\mathcal{A} \boldsymbol{u}=\mathbf{0}$. We have to show that $\boldsymbol{u} \equiv \mathbf{0}$. To this aim, we prove that

$$
\lambda \int_{\mathbb{R}^{d}}|\boldsymbol{u}|^{2}|\boldsymbol{u}|_{\varepsilon}^{p-2} d x \leq 0, \quad p \in(1, \infty) .
$$

Once formula (3.26) is proved, the claim follows easily letting $\varepsilon$ tend to 0 . The argument used to prove (3.26) is similar to that already used in the proofs of Theorems 2.2 and 3.3. For this reason we just sketch it. Note that

$$
\begin{aligned}
\lambda \int_{\mathbb{R}^{d}}|\boldsymbol{u}|^{2}|\boldsymbol{u}|_{\varepsilon}^{p-2} \zeta_{n}^{2} d x= & -\sum_{k=1}^{m} \int_{\mathbb{R}^{d}} \mathfrak{q}\left(u_{k}\right)|\boldsymbol{u}|_{\varepsilon}^{p-2} \zeta_{n}^{2} d x-(p-2) \int_{\mathbb{R}^{d}} \mathfrak{q}\left(|\boldsymbol{u}|_{\varepsilon}\right)|\boldsymbol{u}|_{\varepsilon}^{p-2} \zeta_{n}^{2} d x \\
& -\int_{\mathbb{R}^{d}} \mathfrak{q}\left(|\boldsymbol{u}|_{\varepsilon}, \zeta_{n}^{2}\right)|\boldsymbol{u}|_{\varepsilon}^{p-1} d x-\frac{1}{p} \sum_{i=1}^{d} \int_{\mathbb{R}^{d}}\left\langle B^{i} \boldsymbol{u}, \boldsymbol{u}\right\rangle|\boldsymbol{u}|_{\varepsilon}^{p-2} D_{i} \zeta_{n}^{2} d x \\
& +\frac{p-2}{p} \int_{\mathbb{R}^{d}} \sum_{i=1}^{d}\left\langle B^{i} D_{i} \boldsymbol{u}, \boldsymbol{u}\right\rangle|\boldsymbol{u}|_{\varepsilon}^{p-2} \zeta_{n}^{2} d x \\
& +\frac{2-p}{p} \sum_{i=1}^{d} \int_{\mathbb{R}^{d}}\left\langle B^{i} \boldsymbol{u}, \boldsymbol{u}\right\rangle|\boldsymbol{u}|_{\varepsilon}^{p-3} \zeta_{n}^{2} D_{i}|\boldsymbol{u}|_{\varepsilon} d x \\
& -\int_{\mathbb{R}^{d}}\left[p^{-1}\langle(\operatorname{div} B) \boldsymbol{u}, \boldsymbol{u}\rangle+\langle V \boldsymbol{u}, \boldsymbol{u}\rangle\right]|\boldsymbol{u}|_{\varepsilon}^{p-2} \zeta_{n}^{2} d x
\end{aligned}
$$

where $\zeta_{n}=\zeta\left(n^{-1} \log \psi\right)$ and $\zeta:[0, \infty) \rightarrow[0,1]$ is a smooth function such that $\zeta(s)=1$ if $s \in[0,1]$ and $\zeta(s)=0$ if $s \geq 2$. To ease the notation, we denote by $\mathscr{I}_{j}, j=1, \ldots, 5$ the last five integral terms in the right-hand side of (3.27). We estimate

$$
\left|\mathscr{I}_{1}\right| \leq \varepsilon_{0} \int_{\mathbb{R}^{d}} \mathfrak{q}\left(|\boldsymbol{u}|_{\varepsilon}\right)|\boldsymbol{u}|_{\varepsilon}^{p-2} \zeta_{n}^{2} d x+\frac{1}{\varepsilon_{0}} \int_{B(0, r)} \mathfrak{q}\left(\zeta_{n}\right)|\boldsymbol{u}|_{\varepsilon}^{p} d x
$$

for every $\varepsilon_{0}>0$, where the ball $B(0, r)$ contains the support of function $\zeta$, and, using Hypothesis 3.1(iv) we obtain that

$$
\begin{aligned}
\left|\mathscr{I}_{2}\right| & \leq 2 \frac{m \kappa}{p} \int_{\mathbb{R}^{d}} \sqrt{v} \sqrt{\mathfrak{q}\left(\zeta_{n}\right)}|\boldsymbol{u}|^{2}|\boldsymbol{u}|_{\varepsilon}^{p-2} \zeta_{n} d x \\
& \leq \varepsilon_{2} \int_{\mathbb{R}^{d}} v|\boldsymbol{u}|^{2}|\boldsymbol{u}|_{\varepsilon}^{p-2} \zeta_{n}^{2} d x+\frac{m^{2} \kappa^{2}}{\varepsilon_{2} p^{2}} \int_{\mathbb{R}^{d}} \mathfrak{q}\left(\zeta_{n}\right)|\boldsymbol{u}|^{2}|\boldsymbol{u}|_{\varepsilon}^{p-2} d x
\end{aligned}
$$

for every $\varepsilon_{2}>0$ and

$$
\left|\mathscr{I}_{3}\right| \leq \varepsilon_{1} \kappa \frac{|p-2|}{p} \sum_{k=1}^{m} \int_{\mathbb{R}^{d}} \mathfrak{q}\left(u_{k}\right)|\boldsymbol{u}|_{\varepsilon}^{p-2} \zeta_{n}^{2} d x+\frac{m \kappa}{4 \varepsilon_{1}} \frac{|p-2|}{p} \int_{\mathbb{R}^{d}} v|\boldsymbol{u}|^{2}|\boldsymbol{u}|_{\varepsilon}^{p-2} \zeta_{n}^{2} d x
$$

for every $\varepsilon_{1}>0$. Finally, for every $\varepsilon_{3}>0$, 


$$
\left|\mathscr{I}_{4}\right| \leq \varepsilon_{3} m \kappa \frac{|p-2|}{p} \int_{\mathbb{R}^{d}} \mathfrak{q}\left(|\boldsymbol{u}|_{\varepsilon}\right) \zeta_{n}^{2}|\boldsymbol{u}|_{\varepsilon}^{p-2} d x+\frac{m \kappa}{4 \varepsilon_{3}} \frac{|p-2|}{p} \int_{\mathbb{R}^{d}} v|\boldsymbol{u}|^{2}|\boldsymbol{u}|_{\varepsilon}^{p-2} \zeta_{n}^{2} d x .
$$

In addition,

$$
-\mathscr{I}_{5} \leq\left(\frac{\vartheta}{p}-1\right) \int_{\mathbb{R}^{d}} v|\boldsymbol{u}|^{2}|\boldsymbol{u}|_{\varepsilon}^{p-2} \zeta_{n}^{2} d x
$$

hence we get

$$
\begin{aligned}
\lambda \int_{\mathbb{R}^{d}}|\boldsymbol{u}|^{2}|\boldsymbol{u}|_{\varepsilon}^{p-2} \zeta_{n}^{2} d x \leq & \left(-1+\varepsilon_{1} \kappa \frac{|p-2|}{p}\right) \sum_{k=1}^{m} \int_{\mathbb{R}^{d}} \mathfrak{q}\left(u_{k}\right)|\boldsymbol{u}|_{\varepsilon}^{p-2} \zeta_{n}^{2} d x \\
& +\left(2-p+\varepsilon_{0}+\frac{|p-2|}{p} \kappa m \varepsilon_{3}\right) \int_{\mathbb{R}^{d}} \mathfrak{q}\left(|\boldsymbol{u}|_{\varepsilon}\right)|\boldsymbol{u}|_{\varepsilon}^{p-2} \zeta_{n}^{2} d x \\
& +\left[\varepsilon_{2}-f_{1}\left(\varepsilon_{1}, \varepsilon_{3}\right)\right] \int_{\mathbb{R}^{d}} v|\boldsymbol{u}|^{2}|\boldsymbol{u}|_{\varepsilon}^{p-2} \zeta_{n}^{2} d x \\
& +\left(\frac{1}{\varepsilon_{0}}+\frac{m^{2} \kappa^{2}}{p^{2} \varepsilon_{2}}\right) \int_{B(0, r)} \mathfrak{q}\left(\zeta_{n}\right)|\boldsymbol{u}|_{\varepsilon}^{p} d x,
\end{aligned}
$$

where function $f_{1}$ is defined by (3.14).

Now, we distinguish between the cases $p \geq 2$ and $p \in(1,2)$. In the first case, thanks to condition (3.3), with $\gamma=0$, we can choose $\varepsilon_{i}>0(i=0,1,2,3)$ to ensure that the first three terms in the right-hand side of (3.28) are nonpositive. We refer the reader to Subsection A.3 for further details. Thus, we get

$$
\lambda \int_{\mathbb{R}^{d}}|\boldsymbol{u}|^{2}|\boldsymbol{u}|_{\varepsilon}^{p-2} \zeta_{n}^{2} d x \leq C \int_{B(0, r)} \mathfrak{q}\left(\zeta_{n}\right)|\boldsymbol{u}|_{\varepsilon}^{p} d x
$$

for some positive constant $C$ depending on $m, p, \kappa$. Then, arguing as in the proof of Theorem 2.2, letting $n \rightarrow \infty$ we deduce (3.26).

In the second case, when $p \in(1,2)$ we use estimate (3.8) to deduce that

$$
\begin{aligned}
\lambda \int_{\mathbb{R}^{d}}|\boldsymbol{u}|^{2}|\boldsymbol{u}|_{\varepsilon}^{p-2} \zeta_{n}^{2} d x \leq & {\left[\varepsilon_{0}-g_{1}\left(\varepsilon_{1}, \varepsilon_{3}\right)\right] \sum_{k=1}^{m} \int_{\mathbb{R}^{d}} \mathfrak{q}\left(u_{k}\right)|\boldsymbol{u}|_{\varepsilon}^{p-2} \zeta_{n}^{2} d x } \\
& +\left[\varepsilon_{2}-f_{1}\left(\varepsilon_{1}, \varepsilon_{3}\right)\right] \int_{\mathbb{R}^{d}} v|\boldsymbol{u}|^{2}|\boldsymbol{u}|_{\varepsilon}^{p-2} \zeta_{n}^{2} d x \\
& +\left(\frac{1}{\varepsilon_{0}}+\frac{m^{2} \kappa^{2}}{p^{2} \varepsilon_{2}}\right) \int_{B(0, r)} \mathfrak{q}\left(\zeta_{n}\right)|\boldsymbol{u}|_{\varepsilon}^{p} d x
\end{aligned}
$$

where the function $g_{1}$ is defined by (3.16). Also in this case, using condition (3.2), with $\gamma=0$, we can choose $\varepsilon_{i}>0(i=0,1,2,3)$ to make the first two terms in the right-hand side of (3.29) nonpositive and then we can conclude as in the first case. We refer the reader to Subsection A.4 for further details. 


\section{The semigroup in $C_{b}\left(\mathbb{R}^{d} ; \mathbb{R}^{m}\right)$}

In this section, under suitable assumptions on the coefficients of the operator $\mathcal{A}$, we associate a semigroup $\boldsymbol{T}_{\infty}(t)$ with $\mathcal{A}$ in $C_{b}\left(\mathbb{R}^{d} ; \mathbb{C}^{m}\right)$ that, under the assumptions of Theorem 3.3, is consistent with $\boldsymbol{T}(t)$. Again, we can limit ourselves to considering functions with values in $\mathbb{R}^{m}$.

\section{Hypotheses 4.1}

(i) The coefficients $q_{i j}$ belong to $C_{\mathrm{loc}}^{1+\alpha}\left(\mathbb{R}^{d}\right)$ for $i, j=1, \ldots, d$ and $\langle Q(x) \xi, \xi\rangle \geq \mu_{0}|\xi|^{2}$ for every $x, \xi \in \mathbb{R}^{d}$ and some positive constant $\mu_{0}$; the functions $B^{i}(i=1, \ldots, d)$ satisfy Hypotheses 3.1(ii), (iv), with $\kappa \leq \sqrt{2 m^{-1}}$;

(ii) the function $V$ has entries in $C_{\text {loc }}^{\alpha}\left(\mathbb{R}^{d}\right)$ for some $\alpha \in(0,1)$ and $\langle V(x) \xi, \xi\rangle \geq v(x)|\xi|^{2}$, for every $x, \xi \in \mathbb{R}^{d}$ and some function $v \in C_{\mathrm{loc}}^{\alpha}\left(\mathbb{R}^{d}\right)$ bounded from below by a positive constant $c_{0}$;

(iii) there exists a positive function $\varphi \in C^{2}\left(\mathbb{R}^{d}\right)$ blowing up as $|x| \rightarrow \infty$ such that $\mathcal{A}_{v} \varphi \leq \lambda \varphi$ for some $\lambda>0$, where $\mathcal{A}_{v}=\operatorname{div}(Q D)-v I$ with the function $v$ from Hypothesis 3.1(iv)).

The assumptions on the matrix-valued function $Q$ and on $v$ guarantee that, for every $f \in C_{b}\left(\mathbb{R}^{d}\right)$, there exists a unique function $u \in C_{b}\left([0, \infty) \times \mathbb{R}^{d}\right) \cap C^{1,2}\left((0, \infty) \times \mathbb{R}^{d}\right)$ such that $D_{t} u=\mathcal{A}_{v} u$ on $(0, \infty) \times \mathbb{R}^{d}$ and $u(0, \cdot)=f$ (see [27, 30]). Setting $S(t) f:=u(t, \cdot)$ for every $t \geq 0$, we define a semigroup of bounded operators on $C_{b}\left(\mathbb{R}^{d}\right)$ which satisfies the estimate $\|S(t)\|_{\mathcal{L}\left(C_{b}\left(\mathbb{R}^{d}\right)\right)} \leq e^{-c_{0} t}$ (see, for instance, [5, Proposition 2.2]).

Theorem 4.2 Under Hypotheses 4.1 , for every $f \in C_{b}\left(\mathbb{R}^{d} ; \mathbb{R}^{m}\right)$ the Cauchy problem

$$
\begin{cases}D_{t} \boldsymbol{u}(t, x)=\mathcal{A} \boldsymbol{u}(t, x), & (t, x) \in(0, \infty) \times \mathbb{R}^{d}, \\ {[1 m m] \boldsymbol{u}(0, x)=f(x),} & x \in \mathbb{R}^{d},\end{cases}
$$

admits a unique classical solution $\boldsymbol{u}$, which is bounded in each strip $[0, T] \times \mathbb{R}^{d}$. Moreover,

$$
|\boldsymbol{u}(t, x)| \leq e^{-c_{0} t}\|\boldsymbol{f}\|_{\infty}, \quad(t, x) \in[0, \infty) \times \mathbb{R}^{d} .
$$

Proof The proof of this result is standard. The uniqueness of the locally in time bounded classical solution $\boldsymbol{u}$ follows from estimate

$$
|\boldsymbol{u}(t, x)|^{2} \leq\left(S(t)|\boldsymbol{f}|^{2}\right)(x), \quad(t, x) \in[0, \infty) \times \mathbb{R}^{d},
$$

which can be proved by considering the function $z=|\boldsymbol{u}|^{2}-S(\cdot)|\boldsymbol{f}|^{2}$, which satisfies the inequality $D_{t} z-\mathcal{A}_{v} z \leq 0$ and vanishes when $t=0$. A variant of the classical maximum principle (see [27, Theorem 3.1.3]) implies that $z(t, x) \leq 0$ for every $(t, x) \in(0, \infty) \times \mathbb{R}^{d}$, and the assertion follows.

The existence part can be obtained by compactness, considering the sequence $\left(\boldsymbol{u}_{n}\right) \subset C_{\mathrm{loc}}^{1+\alpha / 2,2+\alpha}((0, \infty) \times B(0, n)) \cap C_{b}([0, \infty) \times B(0, n))$ of functions such that $D_{t} \boldsymbol{u}_{n}=\mathcal{A} \boldsymbol{u}_{n}$ on $(0, \infty) \times B(0, n), \boldsymbol{u}_{n}$ vanishes on $(0, \infty) \times \partial B(0, n)$ and equals function $\boldsymbol{f}$ on $\{0\} \times B(0, n)$ for every $n \in \mathbb{N}$. Each function $\boldsymbol{u}_{n}$ also satisfies the estimate $\left\|\boldsymbol{u}_{n}(t, \cdot)\right\|_{\infty} \leq e^{-c_{0} t}$ for every $t>0$. We refer the reader to [1, Theorem 2.8] for the missing details. 
Thanks to Theorem 4.2 we can associate a semigroup $\boldsymbol{T}_{\infty}(t)$ to $\mathcal{A}$ in $C_{b}\left(\mathbb{R}^{d}, \mathbb{R}^{m}\right)$, by setting $\boldsymbol{T}_{\infty}(\cdot) \boldsymbol{f}:=\boldsymbol{u}$, where $\boldsymbol{u}$ is the solution to Cauchy problem (4.1) provided by Theorem 4.2. Clearly, $\left\|\boldsymbol{T}_{\infty}(t)\right\|_{\mathcal{L}\left(C_{b}\left(\mathbb{R}^{d} ; \mathbb{R}^{m}\right)\right)} \leq e^{-c_{0} t}$ for every $t>0$. This semigroup can be easily extended to $C_{b}\left(\mathbb{R}^{d} ; \mathbb{C}^{m}\right)$ in a straightforward way.

In order to show that the semigroups $\boldsymbol{T}_{p}(t)\left(p \geq p_{0}\right)$ are consistent also with $\boldsymbol{T}_{\infty}(t)$ on $C_{b}\left(\mathbb{R}^{d} ; \mathbb{R}^{m}\right)$ and, hence, on $C_{b}\left(\mathbb{R}^{d} ; \mathbb{C}^{m}\right)$ we show that the domain $D(\boldsymbol{A})$ of the weak generator of $\boldsymbol{T}_{\infty}(t)$ coincides with the maximal domain of $\mathcal{A}$ in $C_{b}\left(\mathbb{R}^{d} ; \mathbb{R}^{m}\right)$. The notion of weak generator $\boldsymbol{A}$ has been extended to vector-valued elliptic operators with unbounded coefficients in $[2,15]$ mimicking the classical definition of infinitesimal generator of a strongly continuous semigroup. Its domain is the set of all functions $\boldsymbol{u} \in C_{b}\left(\mathbb{R}^{d} ; \mathbb{R}^{m}\right)$ such that the function $t \mapsto t^{-1}\left(\boldsymbol{T}_{\infty}(t) \boldsymbol{u}-\boldsymbol{u}\right)$ is bounded in $(0,1]$ with values in $C_{b}\left(\mathbb{R}^{d} ; \mathbb{R}^{m}\right)$ and it pointwise converges on $\mathbb{R}^{d}$ to a continuous function, which defines $\boldsymbol{A u}$.

Proposition 4.3 Under Hypotheses 4.1, the weak generator of the semigroup $\boldsymbol{T}(t)$ coincides with the realization of the operator $\mathcal{A}$ on $D_{\max }(\mathcal{A})$, where

$$
D_{\max }(\mathcal{A})=\left\{\boldsymbol{u} \in C_{b}\left(\mathbb{R}^{d} ; \mathbb{R}^{m}\right) \cap \bigcap_{1 \leq p<\infty} W_{\mathrm{loc}}^{2, p}\left(\mathbb{R}^{d}, \mathbb{R}^{m}\right): \mathcal{A} \boldsymbol{u} \in C_{b}\left(\mathbb{R}^{d} ; \mathbb{R}^{m}\right)\right\}
$$

Moreover, for every $\lambda>0$ and $\boldsymbol{f} \in C_{b}\left(\mathbb{R}^{d} ; \mathbb{R}^{m}\right)$, the function $R(\lambda) \boldsymbol{f}$ defined by

$$
(R(\lambda) \boldsymbol{f})(x)=\int_{0}^{\infty} e^{-\lambda t}\left(\boldsymbol{T}_{\infty}(t) \boldsymbol{f}\right)(x) d t, \quad x \in \mathbb{R}^{d},
$$

belongs to $D_{\max }(\mathcal{A})$ and solves the equation $\lambda \boldsymbol{u}-\mathcal{A} \boldsymbol{u}=\boldsymbol{f}$.

Proof Taking (4.2) into account and arguing as in the proof of [2, Proposition 2.2] the inclusion $D(\boldsymbol{A}) \subset D_{\max }(\mathcal{A})$ can be easily proved. Thus, $D(\boldsymbol{A})=D_{\max }(\mathcal{A})$ if and only if $\lambda I-\boldsymbol{A}$ is injective on $D_{\max }(\mathcal{A})$. To prove the injectivity of this operator, we fix a function $\boldsymbol{u} \in D_{\max }(\mathcal{A})$ such that $\lambda \boldsymbol{u}-\mathcal{A} \boldsymbol{u}=\mathbf{0}$. A straightforward computation reveals that $2 \lambda|\boldsymbol{u}|^{2}-\mathcal{A}_{v}|\boldsymbol{u}|^{2}=\psi$, where

$$
\psi=-2 \sum_{k=1}^{m} \mathfrak{q}\left(u_{k}\right)+2 \sum_{i=1}^{d}\left\langle B^{i} D_{i} \boldsymbol{u}, \boldsymbol{u}\right\rangle-2\langle V \boldsymbol{u}, \boldsymbol{u}\rangle+v|\boldsymbol{u}|^{2} .
$$

Using Hypotheses 4.1 (note that $\kappa \leq \sqrt{2 m^{-1}}$ ) and estimating $\sum_{i=1}^{d}\left\langle B^{i} D_{i} \boldsymbol{u}, \boldsymbol{u}\right\rangle$ $\leq \sum_{k=1}^{m} \mathfrak{q}\left(u_{k}\right)+\frac{\kappa m^{2}}{4} v|\boldsymbol{u}|^{2}$, we conclude first that $\psi \leq 0$ on $\mathbb{R}^{d}$ and then, applying the maximum principle in [27, Theorem 3.1.6], that $\boldsymbol{u}=\mathbf{0}$.

Proposition 4.4 Suppose that the assumptions of Theorem 3.3 are satisfied, for every $p \geq p_{0}$ and some $p_{0}>1$, as well as Hypotheses 4.1. Assume also that $p \kappa^{2} m-4(p-1)^{2} \leq 0$, if $p \in(1,2)$. Then, for every $f \in C_{b}\left(\mathbb{R}^{d} ; \mathbb{C}^{m}\right) \cap L^{p}\left(\mathbb{R}^{d}, \mathbb{C}^{m}\right), p \geq p_{0}$ and $t>0$ it holds that $\boldsymbol{T}_{p}(t) \boldsymbol{f}=\boldsymbol{T}_{\infty}(t) \boldsymbol{f}$.

Proof As we have already stressed, we can limit ourselves to proving that $\boldsymbol{T}_{p}(t) \boldsymbol{f}=\boldsymbol{T}_{\infty}(t) \boldsymbol{f}$ for every $f \in C_{b}\left(\mathbb{R}^{d} ; \mathbb{R}^{m}\right) \cap L^{p}\left(\mathbb{R}^{d} ; \mathbb{R}^{m}\right)$. We fix $\lambda>0, f \in C_{b}\left(\mathbb{R}^{d} ; \mathbb{R}^{m}\right)$, with compact support, and consider the resolvent equation $\lambda \boldsymbol{u}-\mathcal{A} \boldsymbol{u}=\boldsymbol{f}$. By Theorem 3.3 and Proposition 4.3 the previous equation admits a unique solution $\boldsymbol{u}_{r} \in D\left(\boldsymbol{A}_{r}\right)$, for every $r \in\left[p_{0}, \infty\right)$, and a unique solution $\mathbf{u}_{\infty} \in D_{\max }(\mathcal{A})$. Since $\boldsymbol{u}_{r}$ is the Laplace transform of the semigroup $\boldsymbol{T}_{r}(t)$, 
the consistency of the semigroups $\boldsymbol{T}_{p}(t)$ and $\boldsymbol{T}_{q}(t)$ implies that $\boldsymbol{u}_{p}=\boldsymbol{u}_{q}$ for $p, q \in\left[p_{0}, \infty\right)$. Hence, we can simply write $\boldsymbol{u}$ instead of $\boldsymbol{u}_{p}$, and $\boldsymbol{u} \in W_{\text {loc }}^{2, q}\left(\mathbb{R}^{d} ; \mathbb{R}^{m}\right)$ for every $q \in\left[p_{0}, \infty\right)$, so that it is continuous over $\mathbb{R}^{d}$. To prove that it is also bounded on $\mathbb{R}^{d}$, we fix $r>0$, arbitrarily, and $R>0$ such that $\operatorname{supp}(f) \subset B(0, R)$. Since the operator $\boldsymbol{A}_{q}$ is dissipative, we have the estimate $\|\boldsymbol{u}\|_{L^{q}\left(B(0, r) ; \mathbb{R}^{m}\right)} \leq\|\boldsymbol{u}\|_{q} \leq \lambda^{-1}\|\boldsymbol{f}\|_{L^{q}\left(B(0, R) ; \mathbb{R}^{m}\right)}$. Letting $q$ tend to $\infty$ and using the arbitrariness of $r>0$, we conclude that $\boldsymbol{u}$ is bounded over $\mathbb{R}^{d}$, so that it belongs to $C_{b}\left(\mathbb{R}^{d} ; \mathbb{R}^{m}\right)$. Since $\mathcal{A} \boldsymbol{u}=\lambda \boldsymbol{u}-\boldsymbol{f}$, the function $\mathcal{A} \boldsymbol{u}$ is bounded and continuous over $\mathbb{R}^{d}$. Thus, $\boldsymbol{u} \in D_{\max }(\mathcal{A})$ and the equality $\boldsymbol{u}=\boldsymbol{u}_{\infty}$ follows. Hence,

$$
R\left(\lambda, \boldsymbol{A}_{p}\right) \boldsymbol{f}=R(\lambda) \boldsymbol{f}, \quad \lambda>0 .
$$

Using (4.4) and the uniqueness of the Laplace transform one obtains $\boldsymbol{T}_{p}(t) \boldsymbol{f}=\boldsymbol{T}_{\infty}(t) \boldsymbol{f}$ for every $t>0$. To remove the condition on the support of $f$, we observe that every function $f \in C_{b}\left(\mathbb{R}^{d} ; \mathbb{R}^{m}\right) \cap L^{p}\left(\mathbb{R}^{d} ; \mathbb{R}^{m}\right)$ is the limit in $L^{p}\left(\mathbb{R}^{d} ; \mathbb{R}^{m}\right)$ of a sequence $\left(\boldsymbol{f}_{n}\right) \subset C_{c}^{\infty}\left(\mathbb{R}^{d} ; \mathbb{R}^{m}\right)$ which is bounded in $C_{b}\left(\mathbb{R}^{d} ; \mathbb{R}^{m}\right)$ and converge to $f$ pointwise in $\mathbb{R}^{d}$. Taking the limit as $n$ tends to $\infty$ in the equality $\boldsymbol{T}_{p}(t) \boldsymbol{f}_{n}=\boldsymbol{T}_{\infty}(t) \boldsymbol{f}_{n}$, we complete the proof.

Theorem 4.5 Let the assumptions of Proposition 4.4 be satisfied. Further, assume that the function $v$ belongs to $C^{2}\left(\mathbb{R}^{d}\right)$, blows up at infinity and, in addition, satisfies the inequality $\mathcal{A}_{v} v \leq M$ in $\mathbb{R}^{d}$ for some positive constant $M$, if $p \in(1,2)$. Then, the semigroup $\boldsymbol{T}(t)$ maps $L^{p}\left(\mathbb{R}^{d} ; \mathbb{C}^{m}\right)$ into $L^{q}\left(\mathbb{R}^{d} ; \mathbb{C}^{m}\right)$ for every $t>0$ and $p_{0} \leq p \leq q \leq \infty$. Moreover, for every $T>0$, there exists a positive constant $C=C(T, p, q)$ such that

$$
\|\boldsymbol{T}(t) \boldsymbol{f}\|_{L^{q}\left(\mathbb{R}^{d} ; \mathbb{C}^{m}\right)} \leq C t^{-\frac{d}{2}\left(\frac{1}{p}-\frac{1}{q}\right)}\|\boldsymbol{f}\|_{L^{p}\left(\mathbb{R}^{d} ; \mathbb{C}^{m}\right)}, \quad \boldsymbol{f} \in L^{p}\left(\mathbb{R}^{d} ; \mathbb{C}^{m}\right), \quad t \in(0, T] .
$$

Proof Also in this case we prove the assertion for functions with values in $\mathbb{R}^{m}$. For every $p \in\left[p_{0}, \infty\right)$, we consider the contraction semigroup $S_{p}(t)$ generated by the operator $\mathcal{A}_{v}$, with domain $D_{p}\left(\mathcal{A}_{v}\right):=\left\{u \in L^{p}\left(\mathbb{R}^{d}\right) \mid \mathcal{A}_{0} u, v u \in L^{p}\left(\mathbb{R}^{d}\right)\right\}$, in $L^{p}\left(\mathbb{R}^{d}\right)$ (see [29, Theorem 2.4]). The restriction of the semigroup $S_{2}(t)$ to $L^{2}\left(\mathbb{R}^{d}\right) \cap L^{1}\left(\mathbb{R}^{d}\right)$ can be extended to a contraction $C_{0}$-semigroup $S_{1}(t)$ on $L^{1}\left(\mathbb{R}^{d}\right)$ which is consistent with $S_{p}(t)$ for each $p \in(1, \infty)$. Indeed, fix $f \in L^{2}\left(\mathbb{R}^{d}\right) \cap L^{1}\left(\mathbb{R}^{d}\right)$ and $r>0$. Then, $f \in L^{p}\left(\mathbb{R}^{d}\right)$ for every $1<p<2$ and, since the semigroups $S_{p}(t)$ are consistent, we get

$$
\left\|S_{2}(t) f\right\|_{L^{1}(B(0, r))}=\lim _{p \rightarrow 1^{+}}\left\|S_{p}(t) f\right\|_{L^{p}(B(0, r))} \leq \limsup _{p \rightarrow 1}\|f\|_{L^{p}\left(\mathbb{R}^{d}\right)} \leq\|f\|_{L^{1}\left(\mathbb{R}^{d}\right)} .
$$

By letting $r$ tend to $\infty$, we conclude that the restriction of $S_{2}(t)$ to $L^{2}\left(\mathbb{R}^{d}\right) \cap L^{1}\left(\mathbb{R}^{d}\right)$ extends, by density, to a contraction semigroup $S_{1}(t)$ on $L^{1}\left(\mathbb{R}^{d}\right)$. Moreover, if $f \in C_{c}^{\infty}\left(\mathbb{R}^{d}\right)$, then

$$
S_{1}(t) f-f=S_{p}(t) f-f=\int_{0}^{t} S_{p}(s) \mathcal{A}_{p} f d s, \quad t>0 .
$$

Hence for every $p>1$ it follows that $\left\|S_{1}(t) f-f\right\|_{L^{p}(B(0, r))} \leq t\left\|\mathcal{A}_{v} f\right\|_{p}$. Letting first $p$ tend to 1 and then $r$ tend to $\infty$, we deduce that $S_{1}(t) f$ converges to $f$ in $L^{1}\left(\mathbb{R}^{d}\right)$ as $t$ tends to 0 . By density it follows that $S_{1}(t)$ is a strongly continuous semigroup.

On the other hand, $S_{2}(t)$ is the semigroup associated with the quadratic form

$$
a(f)=\int_{\mathbb{R}^{d}} \mathfrak{q}(f) d x+\int_{\mathbb{R}^{d}} v|f|^{2} d x,
$$


with domain $\quad D(a)=\left\{f \in L^{2}\left(\mathbb{R}^{d}\right) \cap W_{\mathrm{loc}}^{1,2}\left(\mathbb{R}^{d}\right):\left|Q^{1 / 2} \nabla f\right|, v^{1 / 2} f \in L^{2}\left(\mathbb{R}^{d}\right)\right\} . \quad$ Note that $a(f) \geq \min \left\{c_{0}, \mu_{0}\right\}\|f\|_{1,2}$ for every $f \in D(a)$. Since by Nash's inequality $\|f\|_{2}^{2+4 / d} \leq C_{1}\|f\|_{1,2}^{2}\|f\|_{1}^{4 / d}$ for some positive constant $C_{1}$ and every $f \in W^{1,2}\left(\mathbb{R}^{d}\right) \cap L^{1}\left(\mathbb{R}^{d}\right)$, we easily obtain that $\|f\|_{2}^{2+4 / d} \leq K a(f)\|f\|_{1}^{4 / d}$ for some constant $K>0$, and therefore $S_{1}(t)$ is bounded from $L^{1}\left(\mathbb{R}^{d}\right)$ into $L^{2}\left(\mathbb{R}^{d}\right)$ (see [14, Theorem 2.4.6]). By observing that $\mathcal{A}_{v}$ is self-adjoint, the usual duality argument proves that $S_{1}(t)$ is bounded from $L^{2}\left(\mathbb{R}^{d}\right)$ into $L^{\infty}\left(\mathbb{R}^{d}\right)$ and, by applying the semigroup law, it follows that $S_{1}(t)$ is bounded from $L^{1}\left(\mathbb{R}^{d}\right)$ into $L^{\infty}\left(\mathbb{R}^{d}\right)$.

Next, we claim that

$$
\left|\left(\boldsymbol{T}_{\infty}(t) \boldsymbol{f}\right)(x)\right|^{p} \leq\left(S(t)|\boldsymbol{f}|^{p}\right)(x), \quad(t, x) \in(0, \infty) \times \mathbb{R}^{d},
$$

for every $f \in C_{b}\left(\mathbb{R}^{d} ; \mathbb{R}^{m}\right)$. Using the fact that $S_{1}(t)$ is bounded from $L^{1}\left(\mathbb{R}^{d}\right)$ into $L^{\infty}\left(\mathbb{R}^{d}\right)$ together with (4.6) it is immediate to check that $\boldsymbol{T}_{p}(t)$ maps $L^{p}\left(\mathbb{R}^{d} ; \mathbb{R}^{m}\right)$ into $L^{\infty}\left(\mathbb{R}^{d} ; \mathbb{R}^{m}\right)$ for every $p \geq p_{0}$ and (4.5) follows in this case. Finally, applying Riesz-Thorin interpolation theorem, we conclude the proof.

So, let us prove the claim. We first consider the case $p \in(1,2)$. A straightforward computation reveals that the function $z_{\varepsilon, p}=|\boldsymbol{u}|_{\varepsilon}^{p}-S(\cdot)\left(|\boldsymbol{f}|^{2}+\varepsilon\right)^{\frac{p}{2}}$, where we have set $\boldsymbol{u}=\boldsymbol{T}_{\infty}(\cdot) \boldsymbol{f}$, is a classical solution to the equation $D_{t} z_{\varepsilon, p}-\mathcal{A}_{v} z_{\varepsilon, p}=\psi_{\varepsilon, p}$ where

$$
\begin{aligned}
\psi_{\varepsilon, p}= & \frac{p(2-p)}{4}|\boldsymbol{u}|_{\varepsilon}^{p-4} \mathfrak{q}\left(|\boldsymbol{u}|^{2}\right)-p|\boldsymbol{u}|_{\varepsilon}^{p-2} \sum_{k=1}^{m} \mathfrak{q}\left(u_{k}\right) \\
& +p|\boldsymbol{u}|_{\varepsilon}^{p-2} \sum_{i=1}^{d}\left\langle B^{i} D_{i} \boldsymbol{u}, \boldsymbol{u}\right\rangle-p|\boldsymbol{u}|_{\varepsilon}^{p-2}\langle V \boldsymbol{u}, \boldsymbol{u}\rangle+v|\boldsymbol{u}|_{\varepsilon}^{p} .
\end{aligned}
$$

Using the hypotheses, the estimate $\mathfrak{q}\left(|\boldsymbol{u}|^{2}\right) \leq 4|\boldsymbol{u}|_{\varepsilon}^{2} \sum_{k=1}^{m} \mathfrak{q}\left(u_{k}\right)$ and Young's inequality, we infer that

$$
\psi_{\varepsilon, p} \leq p(1-p+\sigma)|\boldsymbol{u}|_{\varepsilon}^{p-2} \sum_{k=1}^{m} \mathfrak{q}\left(u_{k}\right)+v|\boldsymbol{u}|_{\varepsilon}^{p-2}\left[|\boldsymbol{u}|^{2}\left(1-p+\frac{p \kappa^{2} m}{4 \sigma}\right)+\varepsilon\right]
$$

for every $\sigma>0$. Choosing $\sigma=p-1$ and using the condition $p \kappa^{2} m-4(p-1)^{2} \leq 0$, we conclude that $D_{t} z_{\varepsilon, p}-\mathcal{A}_{v} z_{\varepsilon, p} \leq \varepsilon v|\boldsymbol{u}|_{\varepsilon}^{p-2}$, whence

$$
z_{\varepsilon, p}(t, x) \leq \int_{0}^{t}\left(S(t-s)\left(\varepsilon v|\boldsymbol{u}|_{\varepsilon}^{p-2}(s, \cdot)\right)\right)(x) d s \leq \varepsilon^{\frac{p}{2}} \int_{0}^{t}(S(t-s) v)(x) d s
$$

for every $t>0$ and $x \in \mathbb{R}^{d}$, where we used the fact that, by the assumption $\mathcal{A}_{v} v \leq M$ in $\mathbb{R}^{d}$ and [27, (proof of) Lemma 4.1.3], we can apply each operator $S(t)$ to function $v$ and $(S(t) v)(x) \leq v(x)+M t$ for every $t \geq 0$ and $x \in \mathbb{R}^{d}$. Letting $\varepsilon \rightarrow 0$ in (4.7), the assertion follows in this case.

Finally, the case $p \in(2, \infty)$ follows easily from estimate (4.3), with $\boldsymbol{u}=\boldsymbol{T}_{\infty}(\cdot) \boldsymbol{f}$, if we recall that $S(t)$ admits an integral representation with a kernel $p:(0, \infty) \times \mathbb{R}^{d} \times \mathbb{R}^{d} \rightarrow \mathbb{R}$ satisfying the condition $\|p(t, x, \cdot)\|_{L^{1}\left(\mathbb{R}^{d}\right)} \leq 1$ for every $t>0$ and $x \in \mathbb{R}^{d}$ (see [27, Theorem 1.2.5]). Hence, by the Hölder's inequality we conclude that $|\boldsymbol{u}(t, \cdot)|^{p} \leq\left(S(t)|\boldsymbol{f}|^{2}\right)^{\frac{p}{2}} \leq S(t)|\boldsymbol{f}|^{p}$ in $\mathbb{R}^{d}$ for every $t>0$. 
Remark 4.6 It is worth noticing that, if the assumptions of Proposition 4.4 are satisfied, with (3.1) replaced by (3.6), to be satisfied by every $\kappa>0$, and $\theta<1$, then the semigroup $\boldsymbol{T}_{p}(t)$ is defined for every $p>1$ (since, by Remark 3.4, the assumptions of Theorem 3.3 are satisfied by every $p>1)$ and estimate (4.5) holds true for every $p, q \in(1, \infty)$, with $p<q$.

\section{Examples}

In this section we provide examples of classes of operators to which the results of this paper can be applied.

Example 5.1 Let $\mathcal{A}$ be the operator defined in (1.1) with

$$
q_{i j}(x)=\left(1+|x|^{2}\right)^{\frac{\alpha}{2}} \delta_{i j}, \quad B^{i}(x)=\left(1+|x|^{2}\right)^{\gamma} e^{\frac{1}{2}\left(1+|x|^{2}\right)^{\beta}} A^{i}
$$

for every $x \in \mathbb{R}^{d}, i, j=1, \ldots, d$ and let $V: \mathbb{R}^{d} \rightarrow \mathbb{R}^{m \times m}$ be a measurable function such that

$$
\langle V(x) \xi, \xi\rangle \geq v(x)|\xi|^{2}, \quad|V(x) \xi| \leq c_{1} v(x)|\xi|
$$

with $v(x):=e^{\left(1+|x|^{2}\right)^{\beta}}$ for $x \in \mathbb{R}^{d}$. Here, $\beta \in(0, \infty), \alpha \in[0,2], \gamma \in[0, \alpha / 4]$ and $A^{i}$ is a symmetric $m \times m$ real-valued matrix for every $i=1, \ldots, d$.

It is easy to check that, for every $\varepsilon>0$, there exists a positive constant $C_{\varepsilon}$ such that

$$
|Q(x)|^{\frac{1}{2}}|\nabla v(x)| \leq \varepsilon v(x)^{\frac{3}{2}}+C_{\varepsilon}, \quad x \in \mathbb{R}^{d} .
$$

Moreover the condition $\alpha \in[0,2]$ ensures that $\langle Q \nabla v, \nabla v\rangle \leq C v^{2} \log ^{2} v$ on $\mathbb{R}^{d}$ for some constant $C>0$. Since $|\nabla Q(y)| \leq C_{*}\left(1+|y|^{2}\right)^{\frac{\alpha-1}{2}}$ for every $y \in \mathbb{R}^{d}$ and $\rho(x)=C_{* *}\left(1+|x|^{2}\right)^{\frac{\alpha}{4}} e^{-\frac{1}{2}\left(1+|x|^{2}\right)^{\beta}}$ for every $x \in \mathbb{R}^{d}$ and some positive constants $C_{*}$ and $C_{* *}$, it follows that

$$
|\nabla Q(y)| \leq C_{*}\left(1+\left(|x|+C_{* *}\left(1+|x|^{2}\right)^{\frac{\alpha}{4}} e^{-\frac{1}{2}\left(1+|x|^{2}\right)^{\beta}}\right)^{2}\right)^{\frac{\alpha-1}{2}}, \quad y \in B(x, \rho(x)) .
$$

Consequently the inequality $\sup _{|x-y| \leq \rho(x)}|\nabla Q(y)| \leq c_{2}\left(1+|x|^{2}\right)^{\frac{\alpha}{2}}(\rho(x))^{-1}$ for every $x \in \mathbb{R}^{d}$ follows immediately, with a positive constant $c_{2}$, since the right-hand side grows faster than the left-hand side as $|x|$ tends to $\infty$.

On the other hand, if we set

$$
A_{0}:=\max _{k \in\{1, \ldots, m\}} \sum_{h=1}^{m}\left(\sum_{i=1}^{d}\left(A_{h k}^{i}\right)^{2}\right)^{\frac{1}{2}},
$$

then, by applying the Cauchy-Schwarz inequality, we get 


$$
\begin{aligned}
\sum_{h, k=1}^{m}\left|\sum_{i=1}^{d} B_{h k}^{i} \eta_{i}^{k}\right|= & \left(1+|x|^{2}\right)^{\gamma} e^{\frac{1}{2}\left(1+|x|^{2}\right)^{\beta}} \sum_{h, k=1}^{m}\left|\sum_{i=1}^{d} A_{h k}^{i} \eta_{i}^{k}\right| \\
& \leq A_{0} \sqrt{v(x)}\left(1+|x|^{2}\right)^{\gamma} \sum_{k=1}^{m}\left|\eta^{k}\right| \\
& \leq A_{0} \sqrt{v(x)}\left(1+|x|^{2}\right)^{\gamma-\frac{\alpha}{4}} \sum_{k=1}^{m}\left\langle Q \eta^{k}, \eta^{k}\right\rangle^{\frac{1}{2}} \\
& \leq A_{0} \sqrt{v(x)} \sum_{k=1}^{m}\left\langle Q \eta^{k}, \eta^{k}\right\rangle^{\frac{1}{2}}
\end{aligned}
$$

for every $x, \eta^{k} \in \mathbb{R}^{d}$. Denote by $\lambda_{A^{i}}, \Lambda_{A^{i}}$, respectively, the minimum and the maximum eigenvalues of $A^{i}$ and let $\lambda \in \mathbb{R}^{d}$ the vector with entries $\lambda_{i}=\left|\lambda_{A^{i}}\right| \vee\left|\Lambda_{A^{i}}\right|(i=1, \ldots, d)$. Then, again by the Cauchy-Schwarz inequality, we can estimate

$$
\begin{aligned}
\langle(\operatorname{div} B(x)) \xi, \xi\rangle & \geq-\left(1+|x|^{2}\right)^{\gamma-1} e^{\frac{1}{2}\left(1+|x|^{2}\right)^{\beta}}\left(2 \gamma+\beta\left(1+|x|^{2}\right)^{\beta}\right) \sum_{i=1}^{d}\left|\left\langle A^{i} \xi, \xi\right\rangle\right|\left|x_{i}\right| \\
& \geq-\left(1+|x|^{2}\right)^{\gamma-1} e^{\frac{1}{2}\left(1+|x|^{2}\right)^{\beta}}\left(2 \gamma+\beta\left(1+|x|^{2}\right)^{\beta}\right)|\xi|^{2}|\lambda||x| \\
& \geq-\vartheta v(x)|\xi|^{2}
\end{aligned}
$$

for every $x \in \mathbb{R}^{d}$ and $\xi \in \mathbb{R}^{m}$, where $\vartheta:=(2 \gamma+\beta)|\lambda| c_{0}$ and

$$
c_{0}:=\max _{x \in \mathbb{R}^{d}}\left\{|x|\left(1+|x|^{2}\right)^{\beta+\gamma-1} e^{-\frac{1}{2}\left(1+|x|^{2}\right)^{\beta}}\right\} .
$$

Therefore, Hypotheses 3.1 are satisfied for every $p>c_{0}(2 \gamma+\beta)|\lambda|$. If we assume further that $4\left(p^{2}-\theta p\right)(p-1)-A_{0}^{2}(2-p)^{2}(\sqrt{m}+m)^{2}>0$, if $\quad p \in(1,2), \quad$ and $4\left(p^{2}-\theta p\right)-(p-2) A_{0}^{2} m(m+p-2)>0$, if $p \geq 2$, then, by Theorem 3.3 (see also Remark 3.4) and [17, Lemma 2.4, Proposition 2.9], which guarantee that

$$
\begin{aligned}
\{u & \left.\in L^{p}\left(\mathbb{R}^{d}\right) \cap W_{\text {loc }}^{2, p}\left(\mathbb{R}^{d}\right): x \mapsto \operatorname{div}\left(\left(1+|x|^{2}\right)^{\alpha / 2} \nabla u(x)\right) \in L^{p}\left(\mathbb{R}^{d}\right)\right\} \\
& =\left\{u \in L^{p}\left(\mathbb{R}^{d}\right) \cap W_{\text {loc }}^{2, p}\left(\mathbb{R}^{d}\right): x \mapsto\left(1+|x|^{2}\right)^{\alpha / 2} D^{2} u(x) \in L^{p}\left(\mathbb{R}^{d}\right)\right\} \\
& =\left\{u \in W^{2, p}\left(\mathbb{R}^{d}\right): x \mapsto\left(1+|x|^{2}\right)^{\alpha / 2} D^{2} u(x) \in L^{p}\left(\mathbb{R}^{d}\right)\right\},
\end{aligned}
$$

the operator

$$
\boldsymbol{A}_{p} \boldsymbol{f}=\operatorname{div}\left(\left(1+|x|^{2}\right)^{\frac{\alpha}{2}} \nabla \boldsymbol{f}\right)+\left(1+|x|^{2}\right)^{\gamma} e^{\frac{1}{2}\left(1+|x|^{2}\right)^{\beta}} \sum_{i=1}^{d} A^{i} D_{i} f-V \boldsymbol{f}
$$

with domain

$$
D\left(\boldsymbol{A}_{p}\right)=\left\{\boldsymbol{f} \in W^{2, p}\left(\mathbb{R}^{d}, \mathbb{C}^{m}\right): x \mapsto\left(1+|x|^{2}\right)^{\alpha / 2} D^{2} \boldsymbol{f}(x),|V \boldsymbol{f}| \in L^{p}\left(\mathbb{R}^{d} ; \mathbb{C}^{m}\right)\right\}
$$

generates an analytic contraction semigroup $\boldsymbol{T}_{p}(t)$ in $L^{p}\left(\mathbb{R}^{d}, \mathbb{C}^{m}\right)$. Moreover, $\boldsymbol{T}_{p}(t) \boldsymbol{f}=\boldsymbol{T}_{q}(t) \boldsymbol{f}$ for all $f \in L^{p}\left(\mathbb{R}^{d}, \mathbb{C}^{m}\right) \cap L^{q}\left(\mathbb{R}^{d}, \mathbb{C}^{m}\right)$ and $q>c_{0}(2 \gamma+\beta)|\lambda|$ satisfying the condition $4\left(q^{2}-\theta q\right)(q-1)-A_{0}^{2}(2-q)^{2}(\sqrt{m}+m)^{2}>0$.

An example of function $V$ satisfying (5.1) for $m=2$ is 


$$
V(x)=\left(\begin{array}{cc}
e^{\left(1+|x|^{2}\right)^{\beta}} & \phi(x) \\
-\phi(x) & e^{\left(1+|x|^{2}\right)^{\beta}}
\end{array}\right)
$$

for every measurable function $\phi$ satisfying $|\phi(x)| \leq e^{\left(1+|x|^{2}\right)^{\beta}}$ for all $x \in \mathbb{R}^{d}$.

Example 5.2 Let $\mathcal{A}$ be the operator defined in Example 5.1 with a potential matrix $V$ whose entries belong to $C_{\mathrm{loc}}^{\alpha}\left(\mathbb{R}^{d}\right)$ and satisfy (5.1). Assuming further that $A_{0} \leq \sqrt{2 m^{-1}}$ if $p \geq 2$ and $p A_{0}^{2} m-4(p-1)^{2} \leq 0$ if $p \in(1,2)$, all the assumptions in Hypotheses 4.1 are satisfied. Hypothesis 4.1(iii) is satisfied, for instance, by the function $\varphi: \mathbb{R}^{d} \rightarrow \mathbb{R}$, defined by $\varphi(x)=1+|x|^{2}$ for every $x \in \mathbb{R}^{d}$. Indeed, since

$$
\left(\mathcal{A}_{v} \varphi\right)(x)=2 \alpha|x|^{2}\left(1+|x|^{2}\right)^{\frac{\alpha}{2}-1}+2 d\left(1+|x|^{2}\right)^{\frac{\alpha}{2}}-\left(1+|x|^{2}\right) e^{\left(1+|x|^{2}\right)^{\beta}}
$$

diverges to $-\infty$ as $|x| \rightarrow \infty$, clearly we can find a positive constant $\lambda$ such that $\mathcal{A}_{v} \varphi \leq \lambda \varphi$. Further,

$$
\begin{gathered}
\left(\mathcal{A}_{v} v\right)(x)=v(x)\left[2 \beta(\alpha+2 \beta-2)|x|^{2}\left(1+|x|^{2}\right)^{\frac{\alpha}{2}+\beta-2}+4 \beta^{2}|x|^{2}\left(1+|x|^{2}\right)^{\frac{\alpha}{2}+2 \beta-2}\right. \\
\left.+2 \beta d\left(1+|x|^{2}\right)^{\frac{\alpha}{2}+\beta-1}-e^{\left(1+|x|^{2}\right)^{\beta}}\right]=: v(x) \psi(x)
\end{gathered}
$$

for every $x \in \mathbb{R}^{d}$. Since $\psi(x)$ diverges to $-\infty$ as $|x| \rightarrow \infty$, the condition $\mathcal{A}_{v} v \leq M$ in $\mathbb{R}^{d}$ is satisfied too. Thus, Proposition 4.4 can be applied to deduce that $\boldsymbol{T}_{p}(t) \boldsymbol{f}=\boldsymbol{T}_{\infty}(t) \boldsymbol{f}$ for every $\boldsymbol{f} \in L^{p}\left(\mathbb{R}^{d} ; \mathbb{C}^{m}\right) \cap C_{b}\left(\mathbb{R}^{d} ; \mathbb{C}^{m}\right)$.

Suppose that $(2 \gamma+\beta)|\lambda| c_{0}<1$ (see Example 5.1) and $\gamma<\alpha / 4$. Then the semigroup $\boldsymbol{T}_{p}(t)$ exists for every $p \in(1, \infty)$ and all the semigroups are consistent.

Finally, Remark 4.6 infers that $\boldsymbol{T}(t)$ maps $L^{p}\left(\mathbb{R}^{d} ; \mathbb{C}^{m}\right)$ into $L^{q}\left(\mathbb{R}^{d} ; \mathbb{C}^{m}\right)$ for every $t>0$ and $1<p \leq q \leq \infty$ and estimate (4.5) holds true.

\section{Appendix A}

\section{Derivation of condition (3.2)}

We need to compute the supremum of the function $f_{2}$ in (3.20) in the set $\Omega=\left\{\left(\varepsilon_{1}, \varepsilon_{2}, \varepsilon_{3}, \varepsilon_{4}\right) \in(0, \infty)^{4}: g_{2}\left(\varepsilon_{1}, \varepsilon_{2}, \varepsilon_{3}, \varepsilon_{4}\right)>0\right\}$, where $g_{2}$ is defined by (3.23). To simplify the notation, we write $f_{2}\left(\varepsilon_{1}, \varepsilon_{2}, \varepsilon_{3}, \varepsilon_{4}\right)=A-B \varepsilon_{1}^{-1}-C \varepsilon_{2}^{-1}-D \varepsilon_{3}^{-1}-D \varepsilon_{4}^{-1}$ and $g_{2}\left(\varepsilon_{1}, \varepsilon_{2}, \varepsilon_{3}, \varepsilon_{4}\right)=E-\varepsilon_{1}-F \varepsilon_{2}-G \varepsilon_{3}-H \varepsilon_{4}$, where

$$
\begin{aligned}
& A=1-\frac{\theta}{p}-\frac{p-1}{p} \kappa m \gamma, \quad B=\frac{\gamma^{2}}{4}, \quad C=\gamma^{2} \frac{|p-2|}{4}, \quad D=\frac{\kappa m|p-2|}{4 p}, \\
& E=p-1, \quad F=2-p, \quad G=\kappa \frac{2-p}{p}, \quad H=\frac{2-p}{p} \kappa m \text {. }
\end{aligned}
$$

From the constraint $g_{2}\left(\varepsilon_{1}, \varepsilon_{2}, \varepsilon_{3}, \varepsilon_{4}\right)>0$, we deduce that $\varepsilon_{1}<E-F \varepsilon_{2}-G \varepsilon_{3}-H \varepsilon_{4}$. Therefore, taking also into account that $\varepsilon_{1}>0$, we deduce that

$$
f_{2}\left(\varepsilon_{1}, \varepsilon_{2}, \varepsilon_{3}, \varepsilon_{4}\right)<\tilde{f}_{2}\left(\varepsilon_{2}, \varepsilon_{3}, \varepsilon_{4}\right):=A-\frac{B}{E-F \varepsilon_{2}-G \varepsilon_{3}-H \varepsilon_{4}}-\frac{C}{\varepsilon_{2}}-\frac{D}{\varepsilon_{3}}-\frac{D}{\varepsilon_{4}}
$$

for every $\left(\varepsilon_{1}, \varepsilon_{2}, \varepsilon_{3}, \varepsilon_{4}\right) \in \Omega$. So, we consider the function $\tilde{f}_{2}$ on the set $\widetilde{\Omega}=\left\{\left(\varepsilon_{2}, \varepsilon_{3}, \varepsilon_{4}\right) \in(0, \infty)^{3}: F \varepsilon_{2}+G \varepsilon_{3}+H \varepsilon_{4}<E\right\}$. Since $\widetilde{f}_{2}\left(\varepsilon_{2}, \varepsilon_{3}, \varepsilon_{4}\right)$ diverges to $-\infty$ 
$\underset{\Omega}{\operatorname{as}}\left(\varepsilon_{2}, \varepsilon_{3}, \varepsilon_{4}\right)$ approaches the points of the boundary of $\widetilde{\Omega}$, this function has a maximum in $\widetilde{\Omega}$. Imposing that its gradient vanishes, we obtain the system

$$
\left\{\begin{array} { l } 
{ \varepsilon _ { 2 } = ( E - F \varepsilon _ { 2 } - G \varepsilon _ { 3 } - H \varepsilon _ { 4 } ) \sqrt { \frac { C } { B F } } , } \\
{ \varepsilon _ { 3 } = ( E - F \varepsilon _ { 2 } - G \varepsilon _ { 3 } - H \varepsilon _ { 4 } ) \sqrt { \frac { D } { B G } } , } \\
{ \varepsilon _ { 4 } = ( E - F \varepsilon _ { 2 } - G \varepsilon _ { 3 } - H \varepsilon _ { 4 } ) \sqrt { \frac { D } { B H } } }
\end{array} \Leftrightarrow \left\{\begin{array}{l}
\varepsilon_{2}=\left(E-F \varepsilon_{2}-G \varepsilon_{3}-H \varepsilon_{4}\right) \sqrt{\frac{C}{B F}}, \\
\varepsilon_{3}=\sqrt{\frac{D F}{C G}} \varepsilon_{2}, \\
\varepsilon_{4}=\sqrt{\frac{D F}{C H}} \varepsilon_{2} .
\end{array}\right.\right.
$$

We conclude that the unique stationary point of $\widetilde{f}_{2}$ is the point $\left(\bar{\varepsilon}_{2}, \bar{\varepsilon}_{3}, \bar{\varepsilon}_{4}\right)$, where

$$
\begin{array}{cl}
\bar{\varepsilon}_{2} & =\frac{E \sqrt{\frac{C}{F}}}{\sqrt{B}+\sqrt{C F}+\sqrt{D G}+\sqrt{D H}}, \quad \bar{\varepsilon}_{3}=\frac{E \sqrt{\frac{D}{G}}}{\sqrt{B}+\sqrt{C F}+\sqrt{D G}+\sqrt{D H}}, \\
\bar{\varepsilon}_{4} & =\frac{E \sqrt{\frac{D}{H}}}{\sqrt{B}+\sqrt{C F}+\sqrt{D G}+\sqrt{D H}},
\end{array}
$$

i.e.,

$$
\tilde{f}_{2}\left(\bar{\varepsilon}_{2}, \bar{\varepsilon}_{3}, \bar{\varepsilon}_{4}\right)=A-\frac{1}{E}(\sqrt{B}+\sqrt{C F}+\sqrt{D G}+\sqrt{D H})^{2},
$$

which is the left-hand side of (3.2). Since $f_{2}$ is continuous at the point $\left(\bar{\varepsilon}_{1}, \bar{\varepsilon}_{2}, \bar{\varepsilon}_{3}, \bar{\varepsilon}_{4}\right)$, where $\bar{\varepsilon}_{1}=E-F \bar{\varepsilon}_{2}-G \bar{\varepsilon}_{3}-H \bar{\varepsilon}_{4}$, and $\widetilde{f}_{2}\left(\bar{\varepsilon}_{2}, \bar{\varepsilon}_{3}, \bar{\varepsilon}_{4}\right)=\lim _{\varepsilon_{1} \rightarrow \bar{\varepsilon}_{1}} f_{2}\left(\varepsilon_{1}, \bar{\varepsilon}_{2}, \bar{\varepsilon}_{3}, \bar{\varepsilon}_{4}\right)$, the second line of (A.1) is also the supremum in $\Omega$ of function $f_{2}$.

\section{Derivation of condition (3.3)}

We still need to consider function $f_{2}$ but in the new set $\Omega=\left\{\left(\varepsilon_{1}, \varepsilon_{2}, \varepsilon_{3}, \varepsilon_{4}\right) \in(0, \infty)^{4}\right.$ : $\left.E-E \varepsilon_{1}-F \varepsilon_{3}>0, E-E \varepsilon_{2}-G \varepsilon_{4}>0\right\}$, where now $E=p, F=\kappa(p-2)$ and $G=\kappa m$. Since $0<\varepsilon_{1}<1-E^{-1} F \varepsilon_{3}$ and $0<\varepsilon_{2}<1-E^{-1} G \varepsilon_{4}$, it follows that

$$
f_{2}\left(\varepsilon_{1}, \varepsilon_{2}, \varepsilon_{3}, \varepsilon_{4}\right)<A-\frac{B E}{E-F \varepsilon_{3}}-\frac{C E}{E-G \varepsilon_{4}}-\frac{D}{\varepsilon_{3}}-\frac{D}{\varepsilon_{4}}=: \widehat{f}_{2}\left(\varepsilon_{3}, \varepsilon_{4}\right)
$$

for every $\left(\varepsilon_{1}, \varepsilon_{2}, \varepsilon_{3}, \varepsilon_{4}\right) \in \Omega$. We consider function $\widehat{f}_{2}$ in $\Omega^{\prime}=\left(0, F^{-1} E\right) \times\left(0, G^{-1} E\right)$, which diverges to $-\infty$ as $\left(\varepsilon_{3}, \varepsilon_{4}\right)$ approaches the boundary of $\Omega^{\prime}$. Hence, it has a maximum in $\Omega^{\prime}$, which is attained at the unique stationary point $\left(\bar{\varepsilon}_{3}, \bar{\varepsilon}_{4}\right)$, where

$$
\bar{\varepsilon}_{3}=\sqrt{\frac{D}{F}} \frac{E}{\sqrt{B E}+\sqrt{D F}}, \quad \bar{\varepsilon}_{4}=\sqrt{\frac{D}{G}} \frac{E}{\sqrt{C E}+\sqrt{D G}},
$$

and

$$
\widehat{f}_{2}\left(\bar{\varepsilon}_{3}, \bar{\varepsilon}_{4}\right)=A-B-2 \sqrt{\frac{B D F}{E}}-\frac{D F}{E}-C-2 \sqrt{\frac{C D G}{E}}-\frac{D G}{E},
$$


which is the left-hand side of (3.3). Since $f_{2}$ is continuous at the point $\left(\bar{\varepsilon}_{1}, \bar{\varepsilon}_{2}, \bar{\varepsilon}_{3}, \bar{\varepsilon}_{4}\right)$, where $\bar{\varepsilon}_{1}=1-E^{-1} F \bar{\varepsilon}_{3}, \bar{\varepsilon}_{2}=1-E^{-1} G \bar{\varepsilon}_{4}$ and $\widehat{f}_{2}\left(\bar{\varepsilon}_{3}, \bar{\varepsilon}_{4}\right)=\lim _{\left(\varepsilon_{1}, \varepsilon_{2}\right) \rightarrow\left(\bar{\varepsilon}_{1}, \bar{\varepsilon}_{2}\right)} f_{2}\left(\varepsilon_{1}, \varepsilon_{2}, \bar{\varepsilon}_{3}, \bar{\varepsilon}_{4}\right)$, we conclude that the second line of (A.2) is also the supremum in $\Omega$ of function $f_{2}$.

\section{On inequality (3.28)}

In this subsection, we prove in details that we can choose the parameters $\varepsilon_{i}(i=0, \ldots, 3)$ in such a way that the first three terms in the right-hand side of (3.28) are all nonpositive. Equivalently, we just need to prove that these parameters can be fixed to guarantee that $f_{1}\left(\varepsilon_{1}, \varepsilon_{3}\right)>0,1-\varepsilon_{1} \kappa(p-2) p^{-1} \geq 0$ and $p-\kappa m \varepsilon_{3}>0$. The better choice of $\varepsilon_{1}$ is $\varepsilon_{1}=p[\kappa(p-2)]^{-1}$. Replacing this value in $f_{1}$, we get a new function $\widetilde{f}_{1}$, which is continuous and increasing in the domain $\left(0, p(\kappa m)^{-1}\right)$. Therefore its supremum in the interval $\left(0, p(\kappa m)^{-1}\right)$ is the limit at $p(\kappa m)^{-1}$, which is $1-\frac{\theta}{p}-\frac{\kappa^{2} m(p-2)}{4 p^{2}}(m+p-2)$, and it is positive due to condition (3.3).

\section{On inequality (3.29)}

Here, we prove in details that the free parameters in (3.29) can be chosen to guarantee that the first two terms in the right-hand side of (3.29) are all nonpositive. Of course, we just need to show that we can choose the positive parameters $\varepsilon_{1}$ and $\varepsilon_{3}$ in such a way that $f_{2}\left(\varepsilon_{1}, \varepsilon_{3}\right)$ and $g_{1}\left(\varepsilon_{1}, \varepsilon_{3}\right)$ are both nonnegative. (Here, $f_{2}$ is the same function defined in (3.20), where now $\gamma=0$, and $g_{1}$ is defined by (3.16).) For this purpose, we compute the supremum of the function $f_{2}$ on the set $\Omega=\left\{\left(\varepsilon_{1}, \varepsilon_{3}\right) \in(0, \infty)^{2}: g_{1}\left(\varepsilon_{1}, \varepsilon_{3}\right)>0\right\}$. We set $E=m, G=p(p-1)[(2-p) \kappa]^{-1}$, so that $g_{1}\left(\varepsilon_{1}, \varepsilon_{3}\right)>0$ is equivalent to $\varepsilon_{1}+F \varepsilon_{3}<G$. Therefore, $0<\varepsilon_{1}<G-F \varepsilon_{3}$ and

$$
f_{2}\left(\varepsilon_{1}, \varepsilon_{3}\right) \leq A^{\prime}-\frac{B^{\prime}}{\varepsilon_{3}}-\frac{B^{\prime}}{C^{\prime}-m \varepsilon_{3}}=: \bar{f}_{2}\left(\varepsilon_{3}\right)
$$

for every $\left(\varepsilon_{1}, \varepsilon_{3}\right) \in \Omega$, where

$$
A^{\prime}=1-\frac{\theta}{p}, \quad B^{\prime}=\frac{\kappa m(2-p)}{4 p}, \quad C^{\prime}-\frac{p(p-1)}{(2-p) \kappa} .
$$

We consider function $\bar{f}_{2}$ in the interval $\left(0, m^{-1} C\right)$. Since it diverges to $-\infty$ as $\varepsilon_{3}$ tends to 0 and $m^{-1} C$, function $\bar{f}_{2}$ has a maximum. Computing the first-order derivative of $\bar{f}_{2}$, we easily realize that such a maximum is attained at $\bar{\varepsilon}_{3}=c(m+\sqrt{m})^{-1}$ and its value is

$$
A^{\prime}-\frac{B^{\prime}}{C^{\prime}}(\sqrt{m}+1)^{2}=1-\frac{\theta}{p}-\frac{\kappa^{2}(2-p)^{2}}{4 p^{2}(p-1)}(\sqrt{m}+m)^{2},
$$

which is positive due to condition (3.2).

If we set $\bar{\varepsilon}_{1}=G-F \bar{\varepsilon}_{3}$ and observe that $f_{2}$ is continuous at $\left(\bar{\varepsilon}_{1}, \bar{\varepsilon}_{3}\right)$, then we conclude that $\lim _{\varepsilon_{1} \rightarrow \bar{\varepsilon}_{1}} f_{2}\left(\varepsilon_{1}, \bar{\varepsilon}_{3}\right)=\bar{f}_{2}\left(\bar{\varepsilon}_{3}\right)$. Moreover, $g_{1}\left(\bar{\varepsilon}_{1}, \bar{\varepsilon}_{3}\right)=0$. These two remarks show that we can choose $\varepsilon_{1}, \varepsilon_{3} \in(0, \infty)$ sufficiently small such that $f_{2}\left(\varepsilon_{1}, \varepsilon_{3}\right)>0$ and $g_{1}\left(\varepsilon_{1}, \varepsilon_{3}\right)>0$. 
Acknowledgements The authors express their gratitude to the anonymous referee for the useful comments and suggestions which have improved the readability of the paper.

Funding Open access funding provided by Università del Salento within the CRUI-CARE Agreement.

Open Access This article is licensed under a Creative Commons Attribution 4.0 International License, which permits use, sharing, adaptation, distribution and reproduction in any medium or format, as long as you give appropriate credit to the original author(s) and the source, provide a link to the Creative Commons licence, and indicate if changes were made. The images or other third party material in this article are included in the article's Creative Commons licence, unless indicated otherwise in a credit line to the material. If material is not included in the article's Creative Commons licence and your intended use is not permitted by statutory regulation or exceeds the permitted use, you will need to obtain permission directly from the copyright holder. To view a copy of this licence, visit http://creativecommons.org/licenses/by/4.0/.

\section{References}

1. Addona, D., Angiuli, L., Lorenzi, L., Tessitore, G.: On coupled systems of Kolmogorov equations with applications to stochastic differential games. ESAIM: Control Optim. Calc. Var. 23, 937-976 (2017)

2. Addona, D., Angiuli, L., Lorenzi, L.: Invariant measures for systems of Kolmogorov equations. J. Appl. Anal. Comput. 8, 764-804 (2018)

3. Addona, D., Angiuli, L., Lorenzi, L.: On invariant measures associated to weakly coupled systems of Kolmogorov equations. Adv. Differ. Equ. 24, 137-184 (2019)

4. Albanese, A.A., Lorenzi, L., Mangino, E.: $L^{p}$-uniqueness for elliptic operators with unbounded coefficients in $\mathbb{R}^{N}$. J. Funct. Anal. 256, 1238-1257 (2009)

5. Angiuli, L., Lorenzi, L.: Compactness and invariance properties of evolution operators associated to Kolmogorov operators with unbounded coefficients. J. Math. Anal. Appl. 379, 125-149 (2011)

6. Angiuli, L., Lorenzi, L.: On coupled systems of PDEs with unbounded coefficients. Dyn. PDE 17, 129-163 (2020)

7. Angiuli, L., Lorenzi, L., Mangino, E.M.: On a perturbation of a class of Schrödinger systems in $L^{2}$ spaces. Note Mat. 38, 125-138 (2018)

8. Angiuli, L., Lorenzi, L., Mangino, E.M., Rhandi, A.: On vector-valued Schrödinger operators with unbounded diffusion in $L^{p}$ spaces. J. Evol. Equ. (2020). https://doi.org/10.1007/s00028-020-00607-9

9. Angiuli, L., Lorenzi, L., Pallara, D.: $L^{p}$ estimates for parabolic systems with unbounded coefficients coupled at zero and first order. J. Math. Anal. Appl. 444, 110-135 (2016)

10. Betz, V., Goddard, B.D., Teufel, S.: Superadiabatic transitions in quantum molecular dynamics. Proc. R. Soc. A 465, 3553-3580 (2009)

11. Dall'Ara, G.M.: Discreteness of the spectrum of Schrödinger operators with non-negative matrix-valued potentials. J. Funct. Anal. 268, 3649-3679 (2015)

12. Davies, E.B.: Some norm bounds and quadratic form inequalities for Schrödinger operators. J. Oper. Theory 9, 147-162 (1983)

13. Davies, E.B.: Some norm bounds and quadratic form inequalities for Schrödinger operators II. J. Oper. Theory 12, 177-196 (1984)

14. Davies, E.B.: Heat Kernels and spectral theory, Cambridge tracts in mathematics. Cambridge University Press, Cambridge (1990)

15. Delmonte, S., Lorenzi, L.: On a class of weakly coupled systems of elliptic operators with unbounded coefficients. Milan J. Math. 79, 689-727 (2011)

16. Engel, K.J., Nagel, R.: One-parameter semigroups for linear evolution equations. Springer-Verlag, New York (2000)

17. Fornaro, S., Lorenzi, L.: Generation results for elliptic operators with unbounded diffusion coefficients in $L^{p}$ - and $C_{b}$ - spaces. Discr. Cont. Dyn. Syst. A 18, 747-772 (2007)

18. Gilbarg, D., Trudinger, N.S.: Elliptic partial differential equations of second order. Springer-Verlag, Berlin (2001)

19. Goldstein, J.A.: Semigroups of linear operators and applications. Oxford University Press, New York, Clarendon Press, Oxford (1985)

20. Hansel, T., Rhandi, A.: The Oseen-Navier-Stokes flow in the exterior of a rotating obstacle: the nonautonomous case. J. Reine Angew. Math. 694, 1-26 (2014)

21. Haslinger, F., Helffer, B.: Compactness of the solution operator to $\bar{\partial}$ in weighted $L^{2}$ - spaces. J. Funct. Anal. 243, 679-697 (2007) 
22. Hieber, M., Lorenzi, L., Prüss, J., Rhandi, A., Schnaubelt, R.: Global properties of generalized Ornstein-Uhlenbeck operators on $L^{p}\left(\mathbb{R}^{N}, \mathbb{R}^{N}\right)$ with more than linearly growing coefficients. J. Math. Anal. Appl. 350, 100-121 (2009)

23. Hieber, M., Rhandi, A., Sawada, O.: The Navier-Stokes flow for globally Lipschitz continuous initial data. Res. Inst. Math. Sci. (RIMS) 1, 159-165 (2007)

24. Hieber, M., Sawada, O.: The Navier-Stokes equations in $\mathbb{R}^{n}$ with linearly growing initial data. Arch. Ration. Mech. Anal. 175, 269-285 (2005)

25. Kunze, M., Lorenzi, L., Maichine, A., Rhandi, A.: $L^{p}$-theory for Schrödinger systems. Math. Nachr. 292, 1763-1776 (2019)

26. Kunze, M., Maichine, A., Rhandi, A.: Vector-valued Schrödinger operators on $L^{p}$ spaces. Discr. Cont. Dyn. Syst. Ser. S 13, 1529-1541 (2020)

27. Lorenzi, L.: Analytical methods for Kolmogorov equations. Monographs and research notes in mathematics, 2nd edn. CRC Press, Boca Raton, FL (2017)

28. Maichine, A., Rhandi, A.: On a polynomial scalar perturbation of a Schrödinger system in $L^{p}$-spaces. J. Math. Anal. Appl. 466, 655-675 (2018)

29. Metafune, G., Pallara, D., Prüss, J., Schnaubelt, R.: $L^{p}$-theory for elliptic operators on $\mathbb{R}^{d}$ with singular coefficients. Z. Anal. Anwend. 24, 497-521 (2005)

30. Metafune, G., Pallara, D., Wacker, M.: Feller semigroups on $\mathbb{R}^{N}$. Semigroup Forum 65, 159-205 (2002)

31. Metafune, G., Prüss, J., Rhandi, A., Schnaubelt, R.: $L^{p}$-regularity for elliptic operators with unbounded coefficients. Adv. Differ. Equ. 10, 1131-1164 (2005)

32. Monniaux, S., Prüss, J.: A theorem of the Dore-Venni type for noncommuting operators. Trans. Amer. Math Soc. 349, 4787-4814 (1997)

33. Okazawa, N.: An $L^{p}$ - theory for Schrödinger operators with nonnegative potentials. Math. Soc. Jpn. 36, 675-688 (1984)

34. Prüss, J., Rhandi, A., Schnaubelt, R.: The domain of elliptic operators on $L^{p}\left(\mathbb{R}^{d}\right)$ with unbounded drift coefficients. Houston J. Math. 32, 563-576 (2006)

Publisher's Note Springer Nature remains neutral with regard to jurisdictional claims in published maps and institutional affiliations. 